04

\title{
Черенковские гамма-телескопы: прошлое, настоящее, будущее. Проект ALEGRO
}

\author{
(C) А.М. Быков, ${ }^{1}$ Ф.А. Агаронян, ${ }^{2,3}$ А.М. Красильщиков, ${ }^{1}$ Е.Е. Холупенко, ${ }^{1}$ П.Н. Аруев, ${ }^{1}$ Д.А. Байко, \\ А.А. Богданов, ${ }^{1}$ Г.И. Васильев, ${ }^{1}$ В.В. Забродский, ${ }^{1}$ С.В. Троицкий, ${ }^{4}$ Ю.В. Тубольцев, ${ }^{1}$ \\ А.А. Кожберов, ${ }^{1}$ К.П. Левенфиш, ${ }^{1}$ Ю.В. Чичагов ${ }^{1}$
}

${ }^{1}$ Физико-технический институт им. А.Ф. Иоффре РАН,

194021 Санкт-Петербург, Россия

${ }^{2}$ Dublin Institute of Advanced Studies,

Dublin, Ireland

${ }^{3}$ Max-Planck-Institut für Kernphysik,

69117 Heidelberg, Germany

${ }^{4}$ Институт ядерных исследований РАН,

117312 Москва, Россия

e-mail: eugene@astro.ioffe.ru

(Поступило в Редакцию 29 ноября 2016 г.)

Дан краткий обзор истории развития атмосферных черенковских гамма-телескопов, а также представлен перечень актуальных задач современной астрофизики и фундаментальной физики, которые могут быть решены с помощью этих инструментов. Представлен проект низкопороговой гамма-обсерватории ALEGRO, ключевой задачей которой является исследование космических гамма-источников с высокой статистикой детектируемых фотонов в диапазоне энергий $5-50 \mathrm{GeV}$, в особенности быстропеременных гамма-источников (гамма-транзиентов).

DOI: 10.21883/JTF.2017.06.44501.2117

\section{Введение}

Наряду с радио-, инфракрасной, оптической и рентгеновской астрономией гамма-астрономия является важнейшей областью наблюдательной астрофизики. Современные методы наблюдений космических гамма-квантов позволяют охватить диапазон от 0.1 мегаэлектроновольта $(\mathrm{MeV})$ до десятков тераэлектроновольт (TeV) [1]. Гамма-астрономия позволяет исследовать наиболее высокоэнергичные явления, происходящие в Галактике и за ее пределами, связанные со взрывами звезд на поздних стадиях эволюции (см., например, [2,3]), слиянием звезд, распространением ударных волн, мощными высокоскоростными истечениями, формирующимися в окрестности сверхмассивных черных дыр в ядрах активных галактик (см., например, [4]). Изучение процессов, происходящих в этих физических системах, важно для создания целостной картины эволюции Вселенной и проверки адекватности теоретических моделей физики элементарных частиц в диапазоне энергий выше $100 \mathrm{TeV}$, недостижимых для современных земных ускорителей. Кроме того, в список задач современной гаммаастрономии входит экспериментальная проверка гипотез фундаментальной физики (например, гипотез о природе темной материи [5], квантовой гравитации, в частности, о нарушении лоренц-инвариантности [6] и др.). Эти нерешенные вопросы делают гамма-астрономию одной из наиболее актуальных областей современной астрофизики, а наблюдения гамма-излучения от космических источников - уникальным источником информации о природе этих объектов.

Наблюдения в гамма-диапазоне сегодня могут производиться как космическими (например, INTEGRAL, AGILE, Fermi и др.), так и наземными инструментами (например, H.E.S.S., VERITAS, MAGIC, ARGO-YBJ, TAIGA-HiSCORE и др.). Наибольшей чувствительностью в диапазоне $0.1-100 \mathrm{TeV}$ обладают черенковские гамма-телескопы. Такие телескопы непосредственно регистрируют не исследуемые космические гамма-кванты, а потоки черенковских фотонов - вторичных частиц, представляющих собой одну из компонент широких атмосферных ливней (ШАЛ), инициированных первичными космическими частицами - гамма-квантами и частицами космических лучей (КЛ) - с энергиями несколько гигаэлектроновольт $(\mathrm{GeV})$ и выше при их взаимодействии с атмосферой Земли. Это делает такие телескопы эффективными инструментами для наблюдений гамма-излучения космических источников, что вместе с относительно низкой (по сравнению со спутниковыми инструментами) стоимостью обусловливает актуальность их использования.

В настоящей работе дан краткий обзор истории создания черенковских гамма-телескопов, представлен перечень наиболее актуальных задач современной астрофизики и фундаментальной физики, которые могут быть решены с помощью этих инструментов, а также представлен проект низкопороговой черенковской гаммаобсерватории, идея которой была выдвинута Ф.А. Агароняном в работе [7]. 


\section{1. Краткая история развития черенковских гамма-телескопов}

В разд. 1 кратко изложена история развития черенковских гамма-телескопов, более подробный технический обзор дан в работе Хилласа [8].

В развитии черенковской гамма-астрономии можно выделить несколько этапов. Первые эксперименты, убедительно показавшие возможность наблюдения черенковского излучения ШАЛ от частиц космических лучей высоких энергий, были проведены в 1950-е г. Гэлбрайтом и Джелли [9] в Великобритании и А.Е. Чудаковым и Н.М. Нестеровой [10] в СССР. Успех этих экспериментов и идея о том, что гамма-кванты высоких энергий, так же как и частицы КЛ, должны инициировать в атмосфеpe ШАЛ, стимулировали первые попытки регистрации космического гамма-излучения с помощью черенковской техники в 1960-е г. Для осуществления этих экспериментов были разработаны установки, которые можно считать первыми черенковскими гамма-телескопами: в СССР - на площадке Крымской станции ФИАН [11], в Великобритании - в Организации по исследованию атомной энергии (A.E.R.E.) в Харвелле [12], в США - в обсерватории Mount Hopkins [13]. В ходе экспериментов на этих установках достоверно зарегистрировать гаммакванты от точечных источников не удалось.

Первые наблюдения, позволившие говорить о наличии высокоэнергичного гамма-излучения от конкретных космических источников, были выполнены в конце 1960-х-начале 1970-х годов. На телескопе Дублинской группы (два рефлектора диаметром $90 \mathrm{~cm}$ ) по результатам наблюдений 1966-1967 гг. был зарегистрирован гамма-сигнал от Крабовидной туманности [14], хотя уровень достоверности детектирования оказался ниже $3 \sigma$. Наблюдения Крабовидной туманности проводились также на первом гамма-телескопе обсерватории Mount Hopkins [13], а в дальнейшем - на усовершенствованной модификации дублинской установки, разработанной коллаборацией Университетского колледжа Дублина и A.E.R.E (четыре рефлектора диаметром $90 \mathrm{~cm}$ [15]). На тех же установках проводились наблюдения и других потенциальных гамма-источников (пульсары СР 1133, НР 1506, СР 0950, галактика М87 и др.): для некоторых из них были получены положительные избытки (например, поток гамма-излучения от пульсара HР 1506 наблюдался на уровне достоверности $2.6 \sigma[15])$. Тем не менее все эти наблюдения в лучшем случае проводились на грани чувствительности, и надежные результаты (с уровнем значимости значительно выше $3 \sigma$ ) на этих установках получить не удалось. В СССР начиная с 1969 г. проводились наблюдения ряда источников космического гамма-излучения на телескопе РЧВ-1 (4 рефлектора диаметром $1.5 \mathrm{~m}$, Крымская астрофизическая обсерватория (КрАО)). Некоторые из этих источников были зарегистрированы на пределе чувствительности (например, Cas $\gamma$-1, в дальнейшем идентифицированный как пульсар 4U $0115+63$, и Cas $\gamma-2$ [16]).
Все перечисленные выше инструменты, как и первые установки Чудакова и др. [11] и Джелли и Портеpa [12], фиксировали только факт черенковской вспышки и работали по принципу прохождения источника через поле зрения телескопа вследствие вращения Земли. Поток гамма-излучения от источника определялся по превышению скорости счета над фоновым значением при прохождении источника через поле зрения телескопа. Такие инструменты принято относить к первому поколению черенковских гамма-телескопов. Наиболее полная и корректная версия метода анализа сигналов от указанных инструментов приведена в работе Ли и Ма [17], результаты которой широко используются для анализа наблюдательных данных современных черенковских телескопов. Следует отметить, что при методах наблюдения и анализа, использовавшихся до работы [17], уровень значимости полезного сигнала $3 \sigma$ не может считаться неоспоримым свидетельством регистрации гамма-источника, так как, например, в ряде случаев наблюдались существенные отрицательные значения превышения сигнала над фоном (вплоть до $-2.7 \sigma$, см., например, $[13,15,16])$. Такая ситуация являлась следствием как неточностей, содержащихся в использованных методах анализа наблюдательных данных, так и сложностей учета сопутствующих факторов (например, погодных условий). Таким образом, нельзя утверждать, что телескопам первого поколения удалось надежно зарегистрировать источники космического гамма-излучения. Тем не менее к их достижениям следует отнести, во-первых, установление верхних пределов на величину потоков гамма-излучения, что позволило исключить некоторые модели формирования гамма-излучения в исследуемых источниках (например, [18]) и установить пределы на значения магнитного поля пульсаров (например, [19]), во-вторых, формирование на основе анализа экспериментальных данных списка потенциальных космических гамма-источников, наблюдение которых следовало провести в первую очередь с помощью более совершенных гамма-телескопов.

Одновременно с наблюдениями на черенковских гамма-телескопах первого поколения были разработаны достаточно сложные и эффективные черенковские гамма-телескопы второго поколения: 10-метровый рефлектор Whipple (наблюдения ведутся с 1968 г., см., например, [20]) в обсерватории Mount Hopkins (сейчас Обсерватория им. Фреда Лоуренса Виппла (Fred Lawrence Whipple Observatory)) и ГТ-48 в КрАО (разработка велась с 1973 г., наблюдения ведутся с 1989 г., см., например, [21]). Эти инструменты отличались от телескопов первого поколения тем, что они, во-первых, давали возможность непрерывно отслеживать положение источника гамма-излучения и, во-вторых, позволяли строить изображения вспышек черенковского излучения (т.е. ярких частей ШАЛ). Однако следует отметить, что до 1978 г. детектор, использовавшийся на Whipple, не позволял строить изображение черенковской вспышки, 
a - как и телескопы первого поколения - лишь фиксировал ее факт. После 1978 г. на Whipple использовалась 19-пиксельная, а с 1983 г. - 37-пиксельная камера [22], собранная из отдельных вакуумных фотоумножителей $(Ф Э У)$. Новый метод анализа наблюдательных данных - определение морфологических признаков изображений черенковских вспышек с помощью параметров Хилласа [23] - позволил осуществлять эффективную селекцию событий, вызванных гамма-квантами и частицами КЛ, т.е. фактически разделить полезный и фоновый сигналы. Это позволило резко повысить качество получаемых данных и существенно снизить требования к экспозиции.

Дополнительными факторами, увеличившими эффективность телескопов второго поколения по сравнению с телескопами первого поколения, являлись: значительное увеличение размеров зеркала и использование стереоскопического метода, т.е. наблюдения черенковской вспышки двумя или более инструментами одновременно под разными углами, что позволило значительно повысить эффективность анализа морфологических признаков изображений, более эффективно производить разделение фона и сигнала и значительно улучшить общее угловое разрешение. Одним из наиболее успешных проектов, реализовавших стереоскопический подход, стал совместный проект Германии, Армении и Испании HEGRA [24]. В 1984 г. группа из Ереванского физического института разработала систему пяти относительно маленьких (диаметром около $3 \mathrm{~m}$ ) телескопов для установки вблизи Бюраканской обсерватории. Позднее решение о расположении телескопов было пересмотрено, и они были установлены на Канарских островах (на высоте $2200 \mathrm{~m}$ над уровнем моря, в обсерватории Observatorio del Roque de los Muchachos на о-ве Ла-Пальма), послужив основой системы телескопов HEGRA. Эти пять телескопов имели одинаковые технические характеристики: зеркало площадью $8.5 \mathrm{~m}^{2}$, поле зрения около $4.5 \mathrm{deg}$ и были оснащены камерами из 271 пиксела-ФЭУ. Четыре телескопа были расположены в вершинах квадрата со стороной $100 \mathrm{~m}$, а пятый телескоп располагался в центре этого квадрата. Такие параметры позволяли обсерватории HEGRA регистрировать гамма-излучение в диапазоне энергий первичных частиц выше $1 \mathrm{TeV}$, достигать углового разрешения около $0.1 \mathrm{deg}$ и энергетического разрешения около $15 \%$. C помощью обсерватории HEGRA был получен целый ряд ценных научных результатов. В частности, спектр гамма-излучения Крабовидной туманности был впервые с высоким уровнем достоверности измерен в диапазоне 0.5-80 TeV [25]. Также было надежно зарегистрировано тераэлектроновольтное гамма-излучение от ряда внегалактических объектов [26]. Эти результаты стали убедительным доказательством эффективности использования стереоскопического подхода в черенковской гаммаастрономии. В то же время наибольшим размером зеркала $(10 \mathrm{~m})$ среди всех черенковских телескопов второго поколения обладал одиночный инструмент Whipple, a зеркала стереоскопических обсерваторий имели меньшие размеры и поэтому общий положительный эффект от совместного использования двух указанных подходов в черенковских телескопах второго поколения не был реализован в полной мере.

Помимо упомянутых выше в 1980-хг. было осуществлено еще несколько проектов черенковских гаммателескопов, таких как CAT (Франция [27]), CANGAROO (Япония, Австралия [28]), его значительно более эффективная модификация CANGAROO II (см., например, [29]), ТАСТIC (Индия [30]), серия телескопов Mark 1-6 (Великобритания, с размещением в США, Австралии и на Канарских островах, см., например, [31,32] и ссылки там же), ШАЛОН (Россия [33]) и др. Все эти инструменты, которые принято также относить ко второму поколению черенковских гамма-телескопов, работали в тераэлектроновольтном диапазоне энергий первичных частиц.

Считается, что именно инструменты второго поколения позволили осуществить надежную регистрацию тераэлектроновольтного гамма-излучения от космических источников: в 1989 г. телескоп Whipple зарегистрировал сигнал от Крабовидной туманности на уровне $9 \sigma$ [34]. Одним из выдающихся результатов, полученных с этими телескопами, стало обнаружение тераэлектроновольтного излучения внегалактических источников - блазаров [26,35-37]. Однако за все время работы черенковских гамма-телескопов второго поколения (вплоть до середины 1990-хг.) было открыто лишь около десяти гамма-источников, причем некоторые из них были зарегистрированы на пределе чувствительности. Стала очевидной необходимость создания более совершенных и чувствительных детекторов, что привело к разработке черенковских гамма-телескопов следующего (третьего) поколения.

\section{2. Современное состояние черенковской гамма-астрономии}

В период 2002-2007 гг. были введены в строй первые черенковские гамма-телескопы третьего поколения: H.E.S.S. [38,39], MAGIC [40,41] и VERITAS [42,43]. Телескопы H.E.S.S. и VERITAS представляют собой сложные стереоскопические системы с большой апертурой и высокой степенью детализации изображения: каждая система изначально содержала 4 телескопа диаметром 10-12 m, оснащенные камерами, состоящими из $10^{2}-10^{3}$ пикселей-ФЭУ. Инструмент MAGIC представлял собой одиночный гамма-телескоп с диаметром зеркала $17 \mathrm{~m}$. На момент ввода в строй эти инструменты регистрировали гамма-излучение в диапазоне 0.1-30 TeV. Телескопы MAGIC (Observatorio del Roque de los Muchachos, Ла-Пальма, Канарские острова) и VERITAS (Fred Lawrence Whipple Observatory, Аризона) ведут наблюдение преимущественно за Северной небесной полусферой, в то время как H.E.S.S. 
(плато Khomas Highland, Намибия) преимущественно наблюдает Южную полусферу. С появлением телескопов третьего поколения связан значительный прогресс в черенковской гамма-астрономии: к настоящему времени открыто уже более 175 космических источников тераэлектроновольтного излучения.

При разработке гамма-телескопов третьего поколения был заложен существенный потенциал для развития и модификации этих инструментов (например, предполагалось, что H.E.S.S. будет состоять из 16 телескопов, а VERITAS - из 7 телескопов [44]), и в последние годы этот потенциал начал успешно реализовываться. В 2009 г. был введен в эксплуатацию телескоп MAGIC II $[45,46]$ (также с диаметром зеркала $17 \mathrm{~m}$, но с более совершенной камерой), и на гаммаобсерватории MAGIC были начаты стереоскопические наблюдения. В 2012г. была произведена модификация камеры телескопа MAGIC I, после чего телескопы стали технически идентичными [47]. Эти операции позволили существенно улучшить чувствительность и понизить пороговую энергию наблюдений до $50 \mathrm{GeV}$ [48]. В 2012 г. на обсерватории H.E.S.S. был введен в эксплуатацию телескоп H.E.S.S. II с эффективным диаметром зеркала $28 \mathrm{~m}$. Ввод в эксплуатацию этого инструмента позволил понизить пороговую энергию регистрации гаммасобытий до 20-30 GeV [49].

\section{3. Основные объекты черенковской гамма-астрономии}

Среди астрофизических объектов и явлений, которые исследуются с помощью черенковских гамма-телескопов, можно выделить следующие.

\section{1. Остатки сверхновых звезд и сверхкаверны}

Вспышки сверхновых звезд связаны с конечными стадиями эволюции массивных звезд и эволюции вырожденных звезд-карликов в двойных звездных системах. Исследования сверхновых звезд имеют фундаментальное значение для астрофизики, поскольку гигантское выделение энергии и импульса, а также выброс синтезированных химических элементов в значительной мере определяют процессы звездообразования и эволюцию галактик. Наблюдения подкласса сверхновых типа Іа позволили установить факт ускоренного расширения Вселенной и способствовали введению понятия гипотетической „темной энергии“. Сверхновые являются источниками электромагнитного излучения во всех спектральных диапазонах; взаимодействие материи, выброшенной при вспышке сверхновой, с окружающей межзвездной средой наблюдается на протяжении многих тысяч лет после вспышки в форме остатков сверхновых $(\mathrm{OCH})$, которые являются наиболее вероятными источниками галактических космических лучей.
Детектирование гамма-излучения ОСН в мегаэлектроновольтном диапазоне с орбитальной гамма-обсерваторией INTEGRAL в ряде случаев позволило оценить содержание нестабильных изотопов ${ }^{44} \mathrm{Ti},{ }^{56} \mathrm{Co}$ и др. в выброшенном веществе, которое было сопоставлено с предсказаниями современных моделей взрыва сверхновых (см., например, [50-52]). Детектирование излучения $\mathrm{OCH}$ с энергиями выше $0.1 \mathrm{GeV}$ с помощью орбитальных гамма-телескопов и на энергиях выше $50 \mathrm{GeV}$ с помощью черенковских гамма-телескопов позволило установить наличие в оболочечных OCH (Tycho's SNR, Cas A, Kepler и др.) механизмов ускорения частиц до энергий выше $10 \mathrm{TeV}$ с эффективностью конверсии кинетической энергии выброса в КЛ порядка 10\% и выше. Высокую гамма-светимость демонстрируют ОСН, взаимодействующие с молекулярными облаками (IC 443, $\mathrm{W} 44$ и др.). В отличие от оболочечных ОСН они ярки в гигаэлектроновольтном диапазоне энергий, но, как правило, имеют более мягкие спектры гамма-излучения. Анализ спектров излучения этих остатков на энергиях ниже $1 \mathrm{GeV}$ орбитальными телескопами Fermi и AGILE принес первые надежные свидетельства роли нуклонных процессов гамма-излучения в ОСН. Гамма-излучение остатка сверхновой RX J1713-3946, зарегистрированное черенковским телескопом H.E.S.S. [53] вплоть до энергий $50 \mathrm{TeV}$, имеет спектр с фотонным индексом около 2. Изображение остатка и достаточно жесткий индекс указывают на возможный вклад лептонных процессов гамма-излучения - обратного комптоновского рассеяния фотонов микроволнового излучения электронами и позитронами, ускоренными до энергий порядка $100 \mathrm{TeV}$ в ОСН. Детальные выводы об относительной роли нуклонного и лептонного механизмов излучения ОСН требуют высокой статистики детектируемых фотонов и информации о параметрах межзвездной среды в окрестности ОСН. Такая информация позволит, в частности, сделать выводы о природе и эффективности процессов ускорения КЛ ударными волнами в ОСН (см., например, [54-56]).

Наблюдениями установлено, что формирование массивных звезд-прародителей сверхновых, связанных с коллапсом ядра, происходит в форме скоплений с различной плотностью звезд. Факт пространственной корреляции сверхновых звезд имеет существенное значение для эволюции межзвездной среды галактик. Сверхкаверны, сформированные множественными вспышками сверхновых, могут являться источниками релятивистских частиц и гамма-излучения [57-59]. Недавно были открыты компактные массивные скопления звезд, которые могут быть эффективными источниками как гаммаизлучения, так и нейтрино высоких энергий [60,61]. Наблюдения данных классов источников с высокой статистикой фотонов позволит определить характер нетепловых компонент в сверкавернах и компактных скоплениях молодых массивных звезд. 


\section{2. Гамма-пульсары}

Пульсары - быстро вращающиеся замагниченные нейтронные звезды, которые являются компактными звездными остатками коллапса ядер массивных звезд. Излучение пульсаров с высокостабильным периодом, генерируемое в их магнитосферах, наблюдается во всех диапазонах электромагнитного спектра от радиоволн до гамма-лучей. После десятилетий интенсивных исследований электродинамика магнитосфер пульсаров по-прежнему представляет собой сложную нерешенную проблему. Это обусловлено сложностью процессов формирования и динамики релятивистской электронпозитронной плазмы в сильных магнитных полях с учетом быстрого вращения компактной звезды (наклонного магнитного ротатора), а также эффектов общей теории относительности. Наблюдаемая форма импульса различна в различных диапазонах энергий и может иметь несколько пиков. Модели конверсии энергии вращения и процессов диссипации магнитных полей вне области светового цилиндра, связанных с эффективным ускорением частиц, могут быть проверены посредством детальных наблюдений гамма-излучения с высокой статистикой фотонов.

Наблюдения гамма-излучения пульсара в Крабовидной туманности, выполненные телескопом Fermi/LAT в диапазоне $0.1-20 \mathrm{GeV}$ [62], показали, что усредненный по фазе спектр этого источника может быть описан следующей зависимостью:

$$
\frac{d F}{d E}=F_{0}(E / 1 \mathrm{GeV})^{-\Gamma} \exp \left(-E / E_{c}\right)
$$

где $F_{0}=(2.36 \pm 0.06 \pm 0.15) \cdot 10^{-7} \mathrm{~cm}^{-2} \mathrm{~s}^{-1} \mathrm{GeV}^{-1}$, спектральный индекс $\Gamma=(1.97 \pm 0.02 \pm 0.06)$, и энергия обрезания $E_{c}=(5.8 \pm 0.5 \pm 1.2) \mathrm{GeV}$. Наблюдения показали, что большая часть энергии регистрируемого гаммаизлучения пульсара содержится в пиках импульсов, суммарная длительность которых может составлять до 20\% полной фазы пульсара.

В более жесткой части гамма-диапазона (выше $30-50 \mathrm{GeV}$ ) наблюдения таких пульсаций могут быть осуществлены с помощью черенковских телескопов [63-68]. В частности, совместное рассмотрение данных черенковской обсерватории MAGIC [63] и данных Fermi/LAT [62] показало, что в диапазоне 5-100 GeV усредненный суммарный спектр импульсов Р1 и Р2 пульсара в Крабовидной туманности (для указанного диапазона находящихся в интервалах фазы $[-0.06 ; 0.04]$ и $[0.32 ; 0.43]$ соответственно, где 0 фазы соответствует максимуму импульса P1 на частоте $1.4 \mathrm{GHz})$ может быть описан следующей степенной зависимостью:

$$
\frac{d F}{d E}=F_{0}(E / 10 \mathrm{GeV})^{-\Gamma}
$$

где $F_{0}=(3.0 \pm 0.2) \times 10^{-10} \mathrm{~cm}^{-2} \mathrm{~s}^{-1} \mathrm{GeV}^{-1}$, спектральный индекс $\Gamma=(3.0 \pm 0.1)$. В диапазоне $0.15-1.5 \mathrm{TeV}$ спектр пульсара в Крабовидной туманности в импульce P2 (интенсивность излучения в котором на энергии $150 \mathrm{GeV}$ примерно в 2 раза превосходит интенсивность излучения в главном импульсе Р1) также может быть описан степенной зависимостью [67]:

$$
\frac{d F}{d E}=F_{0}(E / 150 \mathrm{GeV})^{-\Gamma}
$$

где $F_{0}=(2.0 \pm 0.3) \cdot 10^{-14} \mathrm{~cm}^{-2} \mathrm{~s}^{-1} \mathrm{GeV}^{-1}$, спектральный индекс $\Gamma=(2.9 \pm 0.2)$.

\section{3. Пульсарные туманности (плерионы)}

Процессы в окрестности светового цилиндра быстро вращающегося пульсара могут приводить к ускорению релятивистского ветра, уносящего значительную долю энергии торможения магнитного диполя. Пульсарный ветер представляет собой анизотропный поток холодных ультрарелятивистских частиц с лоренц-фактором вплоть до $\sim 10^{4}-10^{6}$, переносящий магнитные поля с плотностью энергии, превышающей плотность энергии покоя частиц ветра. При взаимодействии замагниченного пульсарного ветра с окружающей средой возникает область диссипации ветра, в которой происходят процессы эффективного ускорения ультрарелятивистских частиц. Синхротронное излучение ускоренных релятивистских электронов и позитронов регистрируется в широком диапазоне от радио- до гамма-фотонов. Обратное комптоновское рассеяние релятивистских электронов на различных фотонных полях, в частности на поле фотонов реликтового излучения и инфракрасного фона, приводит к возникновению потока гамма-квантов с энергиями выше $1 \mathrm{GeV}$. Самым известным представителем этого класса объектов является Крабовидная туманность. На энергиях менее $300 \mathrm{GeV}$ ее излучение может быть зарегистрировано как наземными черенковскими телескопами, обладающими большой эффективной площадью, так и орбитальными детекторами AGILE и Fermi/LAT. В диапазоне $0.1-100 \mathrm{GeV}$ спектр излучения Крабовидной туманности хорошо описывается суммой двух степенных законов, соответствующих указанным выше механизмам формирования излучения [62]. Крабовидная туманность, будучи ярким и достаточно стабильным источником во всем диапазоне электромагнитного спектра, используется для калибровки детекторов большого числа телескопов. Тем более важным оказалось недавнее открытие орбитальными телескопами AGILE и Fermi/LAT гигантских вспышек гамма-излучения Крабовидной туманности в диапазоне энергий фотонов от $100 \mathrm{MeV}$ до нескольких $\mathrm{GeV}$ [69]. До настоящего времени не было обнаружено одновременного с гамма-вспышками изменения потока излучения Крабовидной туманности в других диапазонах спектра, что налагает существенные ограничения на модели происхождения гамма-вспышек и теории формирования спектра излучения туманности. Гамма-вспышки в Крабовидной туманности могут быть объяснены как синхротронное излучение ускоренных на 
ударной волне электронов в сильно флуктуирующих магнитных полях в рамках модели А.М. Быкова и др. [70].

Систематические поиски пульсаров и пульсарных туманностей наземными гамма-телескопами привели к открытию более 30 таких гамма-туманностей (по состоянию на июнь 2016 г.). Это одна из самых многочисленных в Галактике популяций доступных наблюдениям источников тераэлектроновольтного диапазона. Наблюдения объектов этого класса в диапазоне энергий выше нескольких десятков $\mathrm{GeV}$ активно ведутся с помощью современных черенковских телескопов [71-74].

В частности, наблюдения области Vela $\mathrm{X}$, содержащей пульсарную туманность Vela, с помощью черенковского телескопа H.E.S.S. выявили наличие протяженного гамма-источника размером более градуса, обладающего в диапазоне до $10 \mathrm{TeV}$ жестким спектром с фотонным индексом около 1.3, который не становится мягче к краям источника [73]. Размер гамма-источника в несколько раз превышает размер рентгеновской туманности, в значительно большей степени совпадая с положением и размером более крупномасштабной радио-туманности вокруг пульсара Vela. B рамках существующих моделей многоволнового излучения этого объекта спектральные и морфологические характеристики гамма-источника Vela X не могут быть объяснены. Черенковские телескопы нового поколения с более высокой чувствительностью и лучшим разрешением позволят уточнить наблюдаемые характеристики тераэлектроновольтного излучения пульсарных туманностей, в частности, измерить пространственно-разрешенные гамма-спектры, которые будут использованы для уточнения моделей генерации и распространения ускоренных частиц в этих объектах. Увеличение чувствительности также приведет к регистрации гамма-излучения внегалактических пульсарных туманностей; к настоящему времени обнаружен только один такой объект - энергичная гамма-туманность N157B, расположенная в Большом Магеллановом облаке [74].

\section{4. Галактические микроквазары}

Микроквазары - двойные системы с компактным объектом (черной дырой или нейтронной звездой), на который происходит аккреция вещества со звездыкомпаньона, приводящая к формированию высокоскоростных истечений (джетов). Исследования этих объектов имеют существенное значение для понимания физики аккреции и физики релятивистских истечений. Кроме того, микроквазары, возможно, играют важную роль в процессе реионизации Вселенной (ионизации межзвездного газа во Вселенной в эпохи, соответствующие космологическому красному смещению $6<z<30)$. От нескольких микроквазаров зарегистрировано излучение в гигаэлектроновольтном и тераэлектроновольтном диапазонах [75-78].

\section{5. Активные ядра галактик}

Активные ядра галактик (АЯГ) - это системы, связанные со сверхмассивными черными дырами в центрах галактик. Для этих объектов характерно гигантское энерговыделение (см., например, [79]). Считается, что их излучение обусловлено аккрецией вещества на сверхмассивные черные дыры. Измерение детальных спектров АЯГ в гамма-диапазоне необходимо для построения количественных моделей физических процессов, происходящих в этих источниках. Черенковские гаммателескопы ведут интенсивные наблюдения АЯГ (например, $[80,81])$. Хотя для большого количества таких объектов удается установить лишь верхние пределы на потоки излучения в диапазоне выше $0.1 \mathrm{TeV}$ [82], это само по себе является важным результатом, поскольку такие пределы позволяют наложить ограничения на модели ускорения частиц и формирования гаммаизлучения АЯГ. Например, на основании наблюдений, выполненных с помощью черенковского гамма-телескопа H.E.S.S., сделан вывод о том, что „центральная машина“ нашей Галактики (сверхмассивная черная дыра Sagittarius $\mathrm{A}^{*}$ ) может ускорять частицы до энергий порядка $1000 \mathrm{TeV}$ [83]. Происхождение таких частиц, наблюдаемых в земной атмосфере как галактические КЛ, на сегодня является одной из нерешенных актуальных проблем астрофизики высоких энергий.

\section{6. Гамма-всплески}

Гамма-всплески - это вспышки гамма-излучения, по-видимому, связанные со взрывами массивных сверхновых звезд или слиянием вырожденных звезд. Гаммавсплески являются одними из наиболее ярких событий во Вселенной. Длительность гамма-всплесков может варьировать от $10 \mathrm{~ms}$ до нескольких минут [84]. За первоначальной гамма-вспышкой обычно следует более продолжительное свечение, наблюдающееся на более низких частотах. Несмотря на значительный прогресс в исследованиях гамма-всплесков, достигнутый в последние годы [85], природа этих источников остается неясной. Гамма-всплески и следующие за ними послесвечения наблюдаются на всех длинах волн от радио- до гамма-диапазона. Однако наблюдения в самой жесткой части спектра (выше $1 \mathrm{GeV}$ ), выполняемые с помощью спутниковых инструментов, крайне редки ввиду недостаточной чувствительности этих телескопов (эффективная площадь гамма-телескопа Fermi/LAT в этом диапазоне составляет менее $1 \mathrm{~m}^{2}$ [86]). С другой стороны, современные наземные черенковские телескопы не детектируют гамма-всплески, потому что в диапазоне выше нескольких десятков $\mathrm{GeV}$ излучение, сопровождающее эти события, существенно ослабевает, а время наблюдения ограничено несколькими десятками секунд. Тем не менее в последнее десятилетие с помощью черенковских гамма-телескопов были осуществлены несколько попыток регистрации гамма-всплесков (при этом орбиталь- 
ные рентгеновские и гамма-телескопы использовались как триггеры), в результате которых были установлены верхние пределы на значения потоков гамма-излучения, сопровождающего гамма-всплески, в диапазоне выше нескольких сотен $\mathrm{GeV}$ [87-89]. Так, в работе [87] получен верхний предел (на уровне 95\%) на интегральный

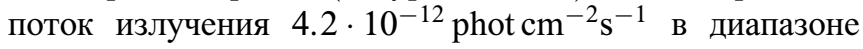
выше $380 \mathrm{GeV}$ от чрезвычайно яркого в рентгеновском диапазоне гамма-всплеска GRB 100621А. Это позволило (на том же уровне достоверности) наложить ограничение на отношение светимостей GRB 100621A в рентгеновском и гамма-диапазонах $L_{X} / L_{V H E}>0.4$, где $L_{X}$ - полная светимость в диапазоне $0.3-10 \mathrm{keV}$, a $L_{V H E}$ - полная светимость в диапазоне $0.38-100 \mathrm{TeV}$. Такое отношение важно для моделирования послесвечения гамма-всплесков и может ограничить модели, в которых предполагается, что лептоны, ответственные за рентгеновское излучение всплеска, также способны сформировать и гамма-излучение сверхвысоких энергий. Физические модели гамма-всплесков основаны на эффективной конверсии мощности релятивистских течений замагниченной плазмы, выделенной в процессе коллапса звезды во вращающуюся черную дыру, в электромагнитное излучение [90]. Следует отметить, что наблюдения наиболее энергичного излучения источников гаммавсплесков исключительно важны, поскольку позволяют впрямую исследовать процессы преобразования энергии, происходящие в так называемой „центральной машине“ всплеска, и таким образом определять природу этих источников.

\section{7. Поиски темной материи}

Ожидается, что выяснение природы темной материи станет одним из важнейших достижений современной физики и астрофизики. Среди многочисленных гипотез о природе темной материи существует ряд таких, для проверки которых могут быть использованы наземные гамма-телескопы. Например, с их помощью можно проверять гипотезы о наличии экзотических частиц темной материи посредством регистрации гамма-излучения, являющегося результатом распада этих частиц, сформированных в ранней Вселенной [91]. В гаммадиапазоне также можно производить поиски излучения, возможно, возникающего при аннигиляции слабовзаимодействующих массивных частиц (WIMP) в гало нашей Галактики. В ряде работ обсуждались указания на то, что диффузное гамма-излучение в узкой линии в направлении центра Галактики или в направлении скопления Coma может быть связано с аннигиляцией легких суперсимметричных WIMP-частиц (см., например, [92,93]). В настоящее время черенковские гаммателескопы осуществляют интенсивные поиски гаммаизлучения от гипотетической аннигиляции частиц темной материи в гало карликовых галактик [94-96]. Результаты этих наблюдений позволили установить верхние пределы на величину сечения такого процесса. Так, по наблюдениям ряда карликовых галактик черенковской гамма-обсерваторией H.E.S.S. [94] был установлен верхний предел $3.9 \cdot 10^{-24} \mathrm{~cm}^{3} \mathrm{~s}^{-1}$ (на уровне достоверности 95\%) на скорость аннигиляции WIMP с массами в диапазоне 1-2 TeV. Совместный анализ данных наблюдений 15 карликовых галактик телескопом Fermi/LAT и наблюдений галактики Segue 1 черенковской гаммаобсерваторией MAGIC [95] позволил установить верхние пределы на скорость аннигиляции WIMP с массами в диапазоне $10 \mathrm{GeV}-100 \mathrm{TeV}$ для различных каналов таких реакций. Типичные значения установленных верхних пределов (уровень достоверности 95\%) составляют от $10^{-26}-10^{-25} \mathrm{~cm}^{3} \mathrm{~s}^{-1}$ (в зависимости от канала) для WIMP с массами $10 \mathrm{GeV}$ до $10^{-22}-10^{-21} \mathrm{~cm}^{3} \mathrm{~s}^{-1}$ (в зависимости от канала) для WIMP с массами $\sim 100 \mathrm{TeV}$. Помимо поисков темной материи, наблюдения гаммаизлучения скоплений галактик или верхние пределы на потоки такого излучения позволяют установить роль нетепловых компонентов в эволюции этих объектов [97].

\section{8. Определение параметров внегалактического инфракрасного фона и поиск аксионоподобных частиц}

Гамма-излучение от далеких источников испытывает интенсивное поглощение при распространении через внегалактическое фоновое излучение [98]. Фоновые инфракрасные фотоны являются основной мишенью для гамма-фотонов. В результате этого взаимодействия рождаются электрон-позитронные пары и происходит выход гамма-излучения из энергетического диапазона, соответствующего чувствительности наземных черенковских гамма-телескопов, в более низкоэнергичный диапазон. Непосредственная оценка диффузного внегалактического инфракрасного фона из наблюдений затруднена из-за трудно учитываемого систематического вклада от зодиакального света и от излучения нашей Галактики, поэтому факты наблюдения высокоэнергичных фотонов тераэлектроновольтного диапазона от удаленных источников были использованы для приближенной оценки сверху величины внегалактического инфракрасного фона (см., например, [99]). Такие оценки с необходимостью основываются на предположениях о характере излучения в источнике, и потому могут рассматриваться как качественные, а не точные количественные. Было обнаружено, что эти оценки сверху на диффузный инфракрасный фон практически совпадают с оценками снизу, получаемыми из подсчета плотности излучения от видимых галактик, а в ряде случаев оказываются и ниже такого фона (см., например, [100]). Проблема несоответствия поглощения гамма-излучения теоретическим моделям инфракрасного фона получила название „кризис ИК-TeV“ (,infrared-TeV crisis“) [101] и привела к пересмотру теоретических нижних пределов. С другой стороны, анализ ансамбля удаленных источников показывает [102,103], что даже использование минимальных моделей приводит к появлению нефизических зависящих 
от расстояния эффектов в реконструированных с учетом поглощения спектрах источников, что указывает на аномальную прозрачность Вселенной для высокоэнергичного гамма-излучения. В качестве объяснения этого эффекта были предложены [104-106] несколько сценариев, в которых фотоны смешиваются с аксионоподобными частицами - гипотетическими псевдоскалярными частицами, взаимодействующими с фотонами так же, как аксион (см., например, обзор [107]). Для подтверждения или опровержения этого объяснения, а также для выбора между сценариями, требуются дальнейшие наблюдения гамма-ярких галактических ядер (блазаров), в том числе наиболее удаленных источников в диапазоне 10-100 GeV [108], которые могут быть выполнены с помощью низкопороговых черенковских гамма-телескопов.

\section{9. Галактический центр}

На основании наблюдений, выполненных с помощью черенковского гамма-телескопа H.E.S.S., в работе [83] сделан вывод о том, что в в окрестности Галактического центра (центральной сверхмассивной черной дыры Sagittarius $\mathrm{A}^{*}$ ) частицы могут ускоряться до энергий $10^{15} \mathrm{eV}$. На это указывает характер спектра гаммаизлучения с отсутствием завала в диапазоне энергий до $100 \mathrm{TeV}$. Поиск галактических источников частиц КЛ с энергиями около и выше $10^{15}$ эВ, наблюдаемых в спектре КЛ, является одной из актуальных нерешенных проблем астрофизики высоких энергий.

На сегодня наиболее полным каталогом зарегистрированных черенковскими гамма-телескопами галактических и внегалактических источников в диапазоне выше $0.1 \mathrm{TeV}$ является каталог TeVCat [109], который по состоянию на июнь 2016 г. содержит 176 таких источников.

Кроме упомянутых выше задач по прямому наблюдению космических гамма-источников и независимому определению внегалактического фонового излучения (см., например, [110]), черенковские гамма- телескопы также позволяют решать такие задачи, как независимое определение интенсивности фона высокоэнергичных электронов (см., например, [111]) и ионов КЛ, проверка моделей квантовой гравитации (см., например, $[66,112,113])$ и некоторые другие.

\section{4. Перспективы развития черенковской гамма-астрономии}

Несмотря на бурное развитие гамма-астрономии в последние десятилетия, ряд актуальных задач, которые могут быть решены с помощью черенковских гаммателескопов, остаются нерешенными, и даже современные системы третьего поколения, такие как H.E.S.S. II и MAGIC II, не обладают характеристиками, достаточными для решения этих задач. В частности, для многих космических источников установлены лишь верхние пределы на величину потоков гамма-излучения, в то время как существование значительного потока гаммаизлучения от них практически не подвергается сомнению [82,87-89]. Это говорит о необходимости создания более мощных инструментов — черенковских гаммателескопов нового (четвертого) поколения.

Важнейшим вопросом черенковской гамма-астрономии является увеличение чувствительности телескопов. Для решения этой проблемы в настоящее время разрабатывается международный проект Cherenkov Telescope Array (CTA): консорциум, включающий 32 страны-участницы [114]. Текущий вариант проекта $[114,115]$ предполагает развертывание двух гаммаобсерваторий: южной (в пустыне Атакама, Чили) и северной (на о. Ла-Пальма, Канарские о-ва, Испания). В рамках проекта СТА будут разработаны три типа телескопов: большой (диаметр зеркала $23 \mathrm{~m}$, чувствительность в диапазоне $20-200 \mathrm{GeV}$, поле зрения около $4.5 \mathrm{deg}$ ), средний (диаметр зеркала $12 \mathrm{~m}$, чувствительность в диапазоне $0.1-10 \mathrm{TeV}$, поле зрения около $7 \mathrm{deg}$ ) и малый (диаметр зеркала $4 \mathrm{~m}$, чувствительность в диапазоне 3-300 TeV, поле зрения около $10 \mathrm{deg}$ ). Южная обсерватория будет состоять из 4 больших, 24-40 средних и 72 малых телескопов и будет размещена на площади около $4 \mathrm{~km}^{2}$. Северная обсерватория будет состоять из 4 больших и 15 средних телескопов и будет размещена на площади около $0.4 \mathrm{~km}^{2}$. За счет размещения множества телескопов на большой площади в проекте СТА будут достигнуты рекордные значения эффективной площади детектирования и чувствительности в диапазоне энергий первичных гамма-квантов выше $100 \mathrm{GeV}$.

Еще одной актуальной проблемой черенковской гамма-астрономии является как можно более существенное снижение пороговой энергии детектирования (в идеальном случае до $2-3 \mathrm{GeV}$ - теоретического предела пороговой энергии черенковских телескопов). В настоящее время не существует инструментов для уверенного наблюдения космических гамма-источников в диапазоне 5-30 GeV. Орбитальные гамма-телескопы не справляются с этой задачей вследствие малой апертуры $\left(\sim 1 \mathrm{~m}^{2}\right)$, а у существующих наземных телескопов не хватает чувствительности для регистрации слишком слабых черенковских вспышек, порождаемых первичными квантами с указанной энергией. Таким образом, даже при наблюдении стационарных источников космического гамма-излучения, которые могут быть осуществлены в течение достаточно длительного времени, не существует надежного перекрытия диапазонов наблюдения орбитальных и наземных инструментов. В то же время измерение спектров космических гамма-источников в широком диапазоне $0.1-1000 \mathrm{GeV}$ представляется чрезвычайно важным по причинам, указанным ниже.

Некоторые типы космических гамма-источников демонстрируют значительное изменение спектральных свойств в диапазоне $1-30 \mathrm{GeV}$. В частности, наблюдения многих пульсаров указывают на наличие экспоненциального падения потока излучения в диапазоне от 1 
до $10 \mathrm{GeV}$ (см., например, [62]), которое может быть описано формулой (1) и согласуется с предсказаниями моделей внутреннего (см., например, [116]) и внешнего зазоров (см., например, [117]) как областей ускорения частиц и генерации гамма-излучения в магнитосфере пульсара в Крабовидной туманности. В то же время моделирование данных [62] с помощью сверхэкспоненциальной формы спектра $\left[\sim \exp \left\{-\left(E / E_{c}\right)^{b}\right\}, b>1\right]$ дало значение $b=(0.89 \pm 0.12 \pm 0.28)$ и позволило исключить значение $b=2$ на уровне достоверности $4.9 \sigma$, фактически показав отсутствие возможностей для описания наблюдаемого спектра с помощью сверхэкспоненциально затухающей функции, предложенной в работе [118].

После исследования [62] решение вопроса о форме спектра пульсара в Крабовидной туманности казалось в достаточной степени ясным. Однако недавние наблюдения этого пульсара с помощью черенковского гаммателескопа MAGIC [63] свидетельствуют о наличии значительного потока излучения на энергиях выше $25 \mathrm{GeV}$ вплоть до энергий $~ 1.5 \mathrm{TeV}$ [67], чего не предсказывала ни одна из рассматривавшихся в тот период моделей. Это стало неожиданностью для специалистов и потребовало модификации стандартных моделей излучения пульсаров [63]. Следует отметить, что в диапазоне $10-30 \mathrm{GeV}$ измерения даже столь яркого объекта, как пульсар в Крабовидной туманности, выполненные с помощью Fermi/LAT и MAGIC, содержат относительно большие экспериментальные ошибки, что обусловлено ухудшением чувствительности обоих инструментов в этом диапазоне по сравнению с чувствительностью в их основных рабочих диапазонах. Это, в частности, выражается в том, что спектральный индекс суммарного спектра $(\mathrm{P} 1+\mathrm{P} 2)$, определенный по данным MAGIC в диапазоне $25-100 \mathrm{GeV}$, составляет $\Gamma_{25}=(3.4 \pm 0.5 \pm 0.3)$ и несколько отличается от значения спектрального индекса $\Gamma_{10}=(3.0 \pm 0.1)$, определенного по совместному анализу данных Fermi/LAT и MAGIC и использованному в формуле (2).

Исследования [62,63] наглядно показывают, что как можно более детальное и корректное измерение пульсарных спектров в диапазоне $5-30 \mathrm{GeV}$ критически важно для выбора правильной модели ускорения частиц и формирования гамма-излучения пульсара. Такое измерение, в свою очередь, требует расширения технических возможностей гамма-астрономии в указанном диапазоне, в частности, ввода в строй новых наземных черенковских гамма-обсерваторий с низким значением пороговой энергии наблюдений.

При наблюдении пульсаров существуют и другие задачи, для решения которых оптимальным инструментом являются черенковские гамма-обсерватории с низким значением пороговой энергии наблюдений. Как предполагается, пучки излучения пульсаров в гамма-диапазоне могут быть значительно шире, чем в радиодиапазоне, и не обязательно перекрываются друг с другом [119]. Это увеличивает шансы на регистрацию быстровращающихся нейтронных звезд в гамма-диапазоне по срав- нению с радио-диапазоном, а также может объяснять наличие гамма-источников в каталогах EGRET и Fermi, не идентифицированных в других диапазонах: значительная доля таких гамма-источников, как предполагается, может быть радиотихими нейтронными звездами (см., например, [120]). Наблюдение пульсирующего излучения от таких объектов в диапазоне энергий выше $5 \mathrm{GeV}$ черенковскими телескопами с их большой площадью детектирования стало бы прямым и неоспоримым доказательством этой гипотезы [121]. Следует отметить, что модель полярных шапок и модель внешнего зазора предсказывают существенно различные значения доли радиотихих нейтронных звезд [122]. Таким образом, достаточно точное измерение доли таких объектов в общем количестве наблюдаемых пульсаров с помощью черенковских гамма-обсерваторий с низким значением пороговой энергии наблюдений стало бы дополнительным аргументом при выборе правильной модели генерации излучения быстровращающимися нейтронными звездами [121].

Дополнительные сложности возникают при наблюдении быстропеременных и вспыхивающих гаммаисточников (например, гамма-всплесков [87-89]), время наблюдения которых ограничено не только техническими возможностями гамма-обсерватории, но и особенностями механизмов излучения. Потоки гамма-излучения от этих источников значительно уменьшаются с ростом энергии регистрируемых фотонов, поэтому целесообразно производить наблюдения в диапазоне 5-30 GeV, где ожидаемые потоки гамма-излучения значительно превосходят потоки излучения в диапазоне выше $30-50 \mathrm{GeV}$, доступном современным черенковским телескопам.

Как в гигаэлектроновольтном, так и в тераэлектроновольтном диапазоне [123] зарегистрировано гаммаизлучение от нескольких микроквазаров, но механизм генерации этого излучения остается неясным, пока не будет исследован промежуточный диапазон $10-100 \mathrm{GeV}$.

Для эффективной регистрации упомянутых выше классов источников в диапазоне $5-30 \mathrm{GeV}$ необходимо создать системы черенковских телескопов с большими зеркалами (диаметром 25-30 m) и расположить их на высотах не менее $4 \mathrm{~km}$ над уровнем моря (см., например, [7]). Детекторы этих телескопов должны обладать более высокой эффективностью детектирования фотонов, чем детекторы существующих телескопов, основанные на использовании традиционных вакуумных фотоумножителей (ФЭУ). Подобное улучшение параметров черенковских телескопов будет направлено не столько на увеличение чувствительности в традиционном для гамма-астрономии высоких энергий в диапазоне 0.1-100 TeV, сколько на снижение пороговой энергии наблюдения до $3-5 \mathrm{GeV}$.

Одним из проектов, направленных на снижение пороговой энергии наблюдений, является проект МАСЕ. Этот проект предполагает установку телескопа с диаметром зеркала $21 \mathrm{~m}$ на высоте $4200 \mathrm{~m}$ [124-126] на 
площадке Индийской астрономической обсерватории в Ханле (Ладакх, Индия). Планируется, что телескоп должен начать работу в 2016 г. Дальнейшее развитие проекта МАСЕ предполагает установку второго телескопа с аналогичными характеристиками в 2018 г. на той же площадке для создания стереоскопической гаммаобсерватории. Однако даже в случае реализации этого проекта в полном объеме, пороговая энергия обсерватории MACE составит не менее $20 \mathrm{GeV}$, и она не сможет в полной мере решить проблему максимально возможного снижения пороговой энергии наблюдений до значений, близких к теоретическому пределу $2-3 \mathrm{GeV}$.

Следует отметить, что попытки создать черенковский телескоп с низкой пороговой энергией $(\sim 10 \mathrm{GeV})$ уже предпринимались ранее в рамках проекта Solar One Gamma Ray Observatory [127], реализованного позднее на базе гелиоэлектростанции Solar Two под названием C.A.C.T.U.S. Однако в рамках этого проекта удалось достичь значения пороговой энергии лишь около $50 \mathrm{GeV}[128]$.

\section{5. Проект гамма-обсерватории ALEGRO}

Для исследования важной, но малоизученной области гамма-диапазона 5-30 GeV в ФТИ им. А.Ф. Иоффе разрабатывается проект черенковской гамма-обсерватории ALEGRO (Atmospheric Low Energy Gamma-Ray Observatory - низкопороговая атмосферная гаммаобсерватория). Энергетический порог этого инструмента (около $5 \mathrm{GeV}$ ) будет существенно ниже, чем у имеющихся черенковских телескопов, что позволит изучить ряд важных особенностей в спектрах стационарных гамма-источников (в частности, экспоненциальные завалы в спектрах пульсаров в диапазоне $1-10 \mathrm{GeV})$, а также исследовать быстропеременное гамма-излучение источников гамма-всплесков и активных ядер галактик, с чувствительностью и временны́м разрешением, существенно лучшими, чем у существующих гамма-обсерваторий. Для достижения этих целей планируется использовать следующие подходы.

1. Обсерватория ALEGRO будет расположена на высоте 4-5 km над уровнем моря. В настоящее время рассматриваются два варианта размещения обсерватории. Первый вариант предусматривает установку телескопов на высоте 4.7-5.3 km в пустыне Атакама (Чили, Аргентина, рис. 1, левая панель). Эта местность обладает уникальным астрономическим климатом. Благодаря строительству современного радиотелескопа LLAMA там уже имеется развитая инфраструктура, что позволит сэкономить значительные средства. Второй вариант, разрабатываемый совместно с Институтом ядерных исследований (ИЯИ) РАН, предусматривает установку телескопов на высоте $3.7 \mathrm{~km}$ в Приэльбрусье,
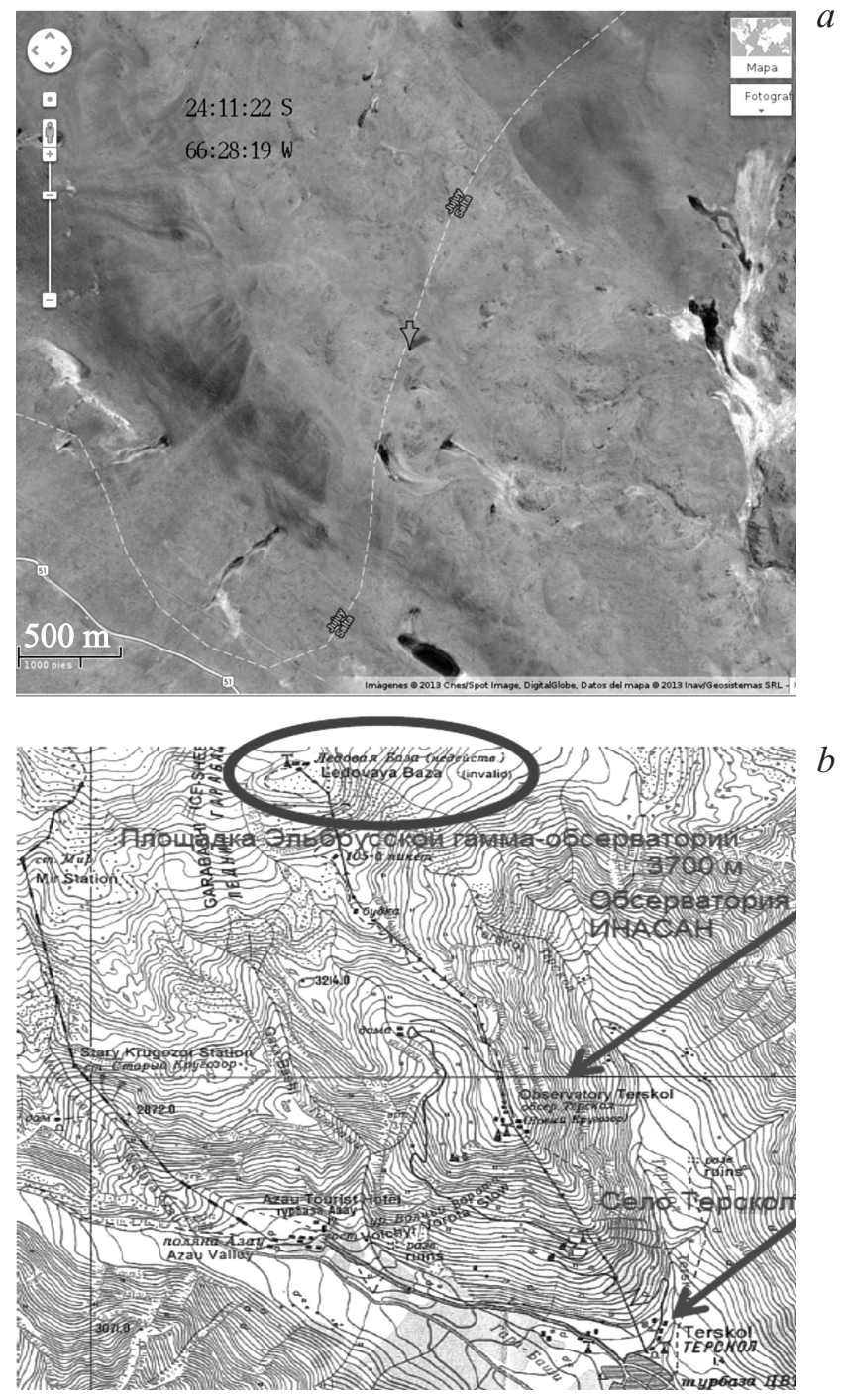

Рис. 1. Карты площадок, где возможно размещение черенковской гамма-обсерватории ALEGRO: $a$ ) площадка в пустыне Атакама (Аргентина), $b$ ) площадка в Приэльбрусье.

в $10 \mathrm{~km}$ от с. Терскол (рабочее название - „Эльбрусская Гамма-Обсерватория“ (ЭГО), рис. 1, правая панель). Наиболее важно то, что на рассматриваемых высотах поверхностная плотность черенковских фотонов (ППЧФ) примерно в 2 раза превышает плотность на высоте $\sim 2 \mathrm{~km}$ (рис. 2), где расположены системы черенковских гамма-телескопов H.E.S.S. и MAGIC. Это само по себе делает регистрацию ШАЛ на высотах около $5 \mathrm{~km}$ значительно более эффективной, чем на высотах около $2 \mathrm{~km}$ при прочих равных параметрах обсерваторий.

2. Вместо традиционных высоковольтных ФЭУ в телескопах ALEGRO будут использованы многопиксельные гейгеровские лавинные фотодиоды (МЛФД), также известные как кремниевые фотоумножители (см., например, [129]). МЛФД способны надежно регистрировать единичные черенковские фотоны за счет внут- 


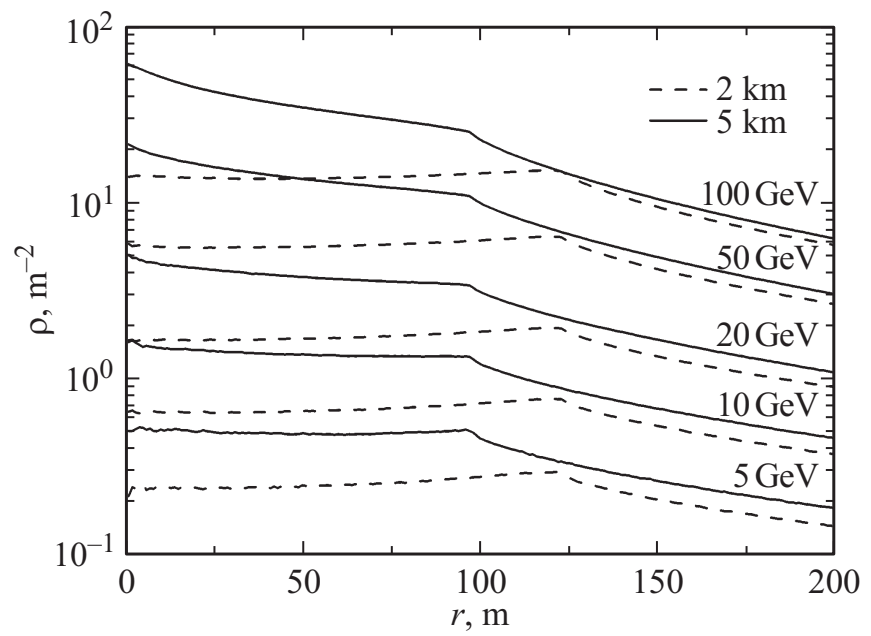

Рис. 2. Зависимость среднего значения поверхностной плотности черенковских фотонов (ППЧФ) от расстояния до оси широкого атмосферного ливня (ШАЛ) для различных энергий первичного гамма-кванта (указаны рядом с кривыми) и высот наблюдения.

реннего бесшумового усиления сигнала на несколько порядков величины, достигаемого за счет образования электронно-дырочной лавины при попадании в детектор оптического фотона. Многопиксельный детектор состоит из множества микроячеек, соединенных параллельно; если на детектор попадает одновременно несколько фотонов, то каждый фотон порождает лавину только в одной микроячейке, а электрический сигнал на выходе будет пропорционален количеству сработавших микроячеек. Численное моделирование позволяет произвести оценку поверхностной плотности черенковских фотонов при энергиях первичных гаммаквантов около $5 \mathrm{GeV}$ (см., например, [7,130]), которая показывает, что МЛФД, используемые как детекторы черенковского излучения, удовлетворяют требованиям полноценного функционирования наземных гамма-телескопов при таких низких энергиях первичных частиц. Основное преимущество МЛФД в сравнении с традиционными вакуумными ФЭУ заключается в том, что применение МЛФД позволяет проводить наблюдения в лунные ночи, что существенно увеличивает экспозиции исследуемых космических источников, а также в высокой квантовой эффективности - 30-40\% вместо 10-20\% (см., например, [131,132]). Кроме этого, МЛФД проще в использовании, требуют значительно меньшего электрического напряжения и мощности. Это существенным образом уменьшает вес детектора и позволяет предъявлять менее жесткие требования к механическим системам телескопа, обеспечивающим размещение детектора в фокусе зеркала и вращение всей конструкции (детектор и зеркало). Единственным существенным недостатком кремниевых фотоумножителей является температурная чувствительность рабочего напряжения, которая требует исполь- зования системы обратной связи с термодатчиками. Следует отметить, что технология такой обратной связи уже прошла апробацию и показала свою высокую эффективность на черенковском телескопе-прототипе FACT [133].

3. Для регистрации как можно большего числа черенковских фотонов в телескопах ALEGRO будут использованы зеркала 30-метрового класса, собранные из отдельных сегментов (см., например, [7]). Несмотря на значительные размеры, у комплекса сохранится возможность наведения на исследуемый участок неба за 20-40 s. В существующих черенковских телескопах используются в основном 12-17-метровые зеркала, детектирующие в 3-6 раз меньше фотонов.

4. ALEGRO будет представлять собой комплекс из четырех телескопов, смонтированных на железнодорожных шасси, аналогично тому, как это реализовано, например, в радиоинтерферометре VLA [134]. В случае невозможности прокладки железнодорожного полотна вследствие особенностей местности будет рассмотрен вариант монтажа всех или нескольких телескопов обсерватории на автомобильных шасси (в качестве тягача может быть использован карьерный самосвал БЕЛАЗ серий 7530-7560 грузоподъемностью 220-360 тонн). Основным преимуществом одновременного использования нескольких телескопов является возможность наблюдения одной и той же атмосферной лавины с нескольких направлений. Эта техника позволяет точно воспроизвести геометрическую картину лавины, а также отличить атмосферные ливни, вызванные гаммаквантами, от фоновых событий, порождаемых космическими лучами. Использование массива черенковских телескопов позволяет на порядок повысить чувствительность по сравнению с наблюдениями на одном телескопе, снижает энергетический порог, а также улучшает угловое и энергетическое разрешения. Подобная схема уже успешно используется в гамма-телескопах H.E.S.S. и VERITAS, однако эти телескопы имеют стационарные точки стояния. Монтаж телескопов обсерватории ALEGRO на железнодорожных или автомобильных шасси позволит изменять пространственную конфигурацию гамма-обсерватории, максимально адаптируя ее для решения конкретных задач. Точность взаимного позиционирования будет обеспечиваться локальными датчиками дистанции. Для обработки и анализа наблюдательных данных обсерватории ALEGRO будет создано оригинальное программное обеспечение, при разработке которого особое внимание будет уделено корректности и эффективности работы анализирующих алгоритмов при энергиях первичных частиц 5-50 GeV.

Следует отметить, что оценочная стоимость проекта ALEGRO в описанной выше конфигурации оказывается более чем на порядок ниже, чем стоимость орбитального гамма-телескопа Fermi, которая составила около 700 миллионов долларов в ценах 2008 г. (см., напримep, [135]). 


\section{6. Моделирование параметров гамма-обсерватории ALEGRO}

В ФТИ им. А.Ф. Иоффе с 2012 г. проводится массированное моделирование черенковских вспышек в широких атмосферных ливнях, вызванных космическими гаммаквантами. Моделируются изображения вспышек в телескопе, разрабатываются алгоритмы быстрой обработки изображений, изучаются сопутствующие атмосферные явления, влияющие на процесс наблюдений. Ведется разработка специализированного пакета программного обеспечения (ПО) для анализа наблюдательных данных будущей гамма-обсерватории (рабочее название ПО „ALEGRO Soft“). К настоящему моменту в рамках этой части проекта выполнены следующие работы.

1) Разработан оригинальный код для моделирования ШАЛ с использованием пакета библиотек элементарных ядерно-физических процессов GEANT [136]. Следует отметить, что разработанный в ФТИ пакет программ обладает рядом качественных преимуществ по сравнению с кодом CORSIKA [137], наиболее широко используемым для моделирования ШАЛ. Так, например, в ALEGRO Soft заложена возможность быстрой, не требующей досконального знания кода, модификации расчетов для различных параметров атмосферы (температуры, плотности, концентрации озона и примесей, величины и направления магнитного поля Земли и т.д.), обеспечено применение самых современных моделей ядерных взаимодействий (они регулярно обновляются в рамках проекта GEANT) и т. п. Одним из наиболее существенных отличий является то, что в коде ALEGRO Soft не предполагается объединения фотонов с близкими значениями волнового вектора в пучки (bunch) для совместной обработки, как это происходит в CORSIKA, a траектория каждого фотона рассчитывается отдельно. Это важно, поскольку метод расчета траекторий фотонов путем объединения их в пучки приводит к значительным искажениям изображений моделируемых черенковских вспышек при энергиях первичных частиц менее $30 \mathrm{GeV}$. Указанные преимущества позволяют использовать ALEGRO Soft для обоснования актуальности, возможности создания и планирования наземной гамма-обсерватории четвертого поколения. Поскольку CORSIKA является наиболее широко используемым в научной практике кодом для моделирования ШАЛ, а его результаты признаны научным сообществом и используются для анализа данных наблюдений на многих современных наземных гамма-телескопах второго и третьего поколений, было проведено сравнение результатов моделирования с помощью ALEGRO Soft с результатами CORSIKA для набора тестовых задач, которое показало, что максимальная разница средних значений поверхностной плотности черенковских фотонов (ППЧФ) не превышает 0.5 стандартных отклонений, что с учетом в разнице используемых численных методов говорит об отличном согласии ALEGRO Soft и CORSIKA при решении тестовых задач для энергий выше $30-50 \mathrm{GeV}$.

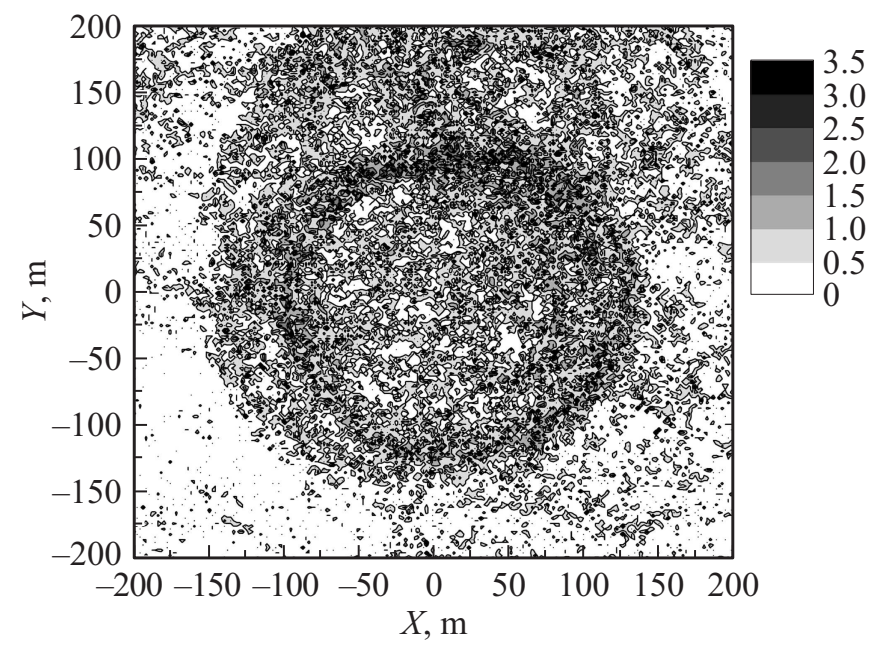

Рис. 3. Распределение интегрированной по спектру поверхностной плотности фотонов черенковской вспышки, вызванной гамма-квантом с энергией $5 \mathrm{GeV}$, на высоте наблюдения $5 \mathrm{~km}$. Цветовая шкала соответствует ППЧФ в единицах photon $/ \mathrm{m}^{2}$.

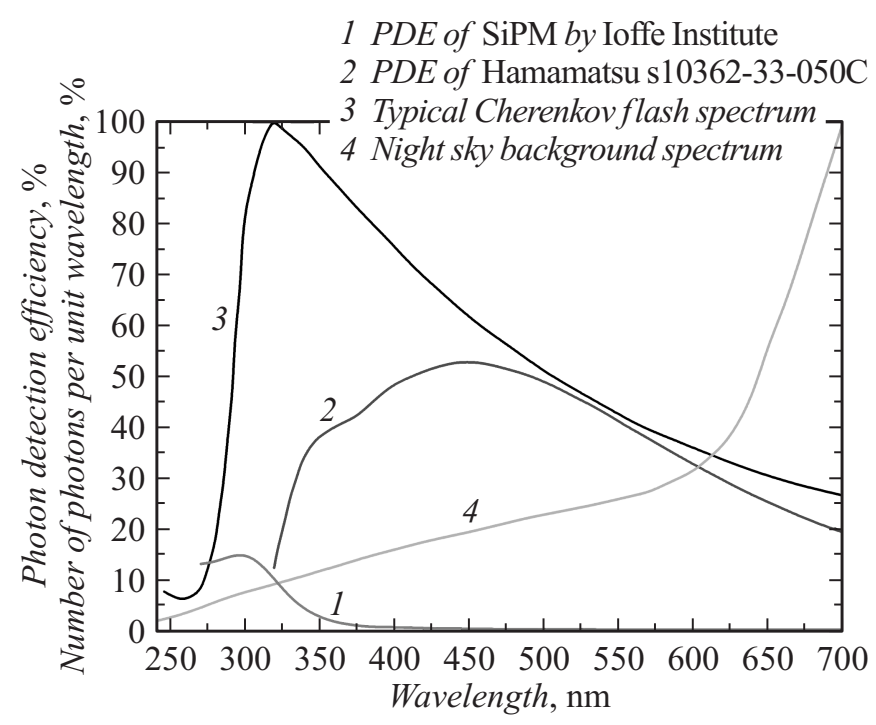

Рис. 4. Модельный спектр черенковского излучения широкого атмосферного ливня (нормированный на 100\% в максимуме (на длине волны $320 \mathrm{~nm}$ ) на высоте $5 \mathrm{~km}$ над уровнем моря, вызванного гамма-событием в атмосфере Земле (кривая 3), а также фоновый спектр ночного неба, нормированный на $100 \%$ на длине волны $700 \mathrm{~nm}$ (кривая 4, расчет основан на данных $[138,139])$, и кривые чувствительности МЛФД производства Hamamatsu (кривая 2, [140]) и ФТИ (кривая 1).

Пример результатов работы ALEGRO Soft представлен на рис. 3, где показана поверхностная плотность фотонов черенковской вспышки от ШАЛ, вызванного вертикально падающим гамма-квантом с энергией $5 \mathrm{GeV}$ как функция координат на высоте наблюдения $5 \mathrm{~km}$. Модельный спектр черенковской вспышки приведен на рис. 4 наряду с фоновым спектром ночного 

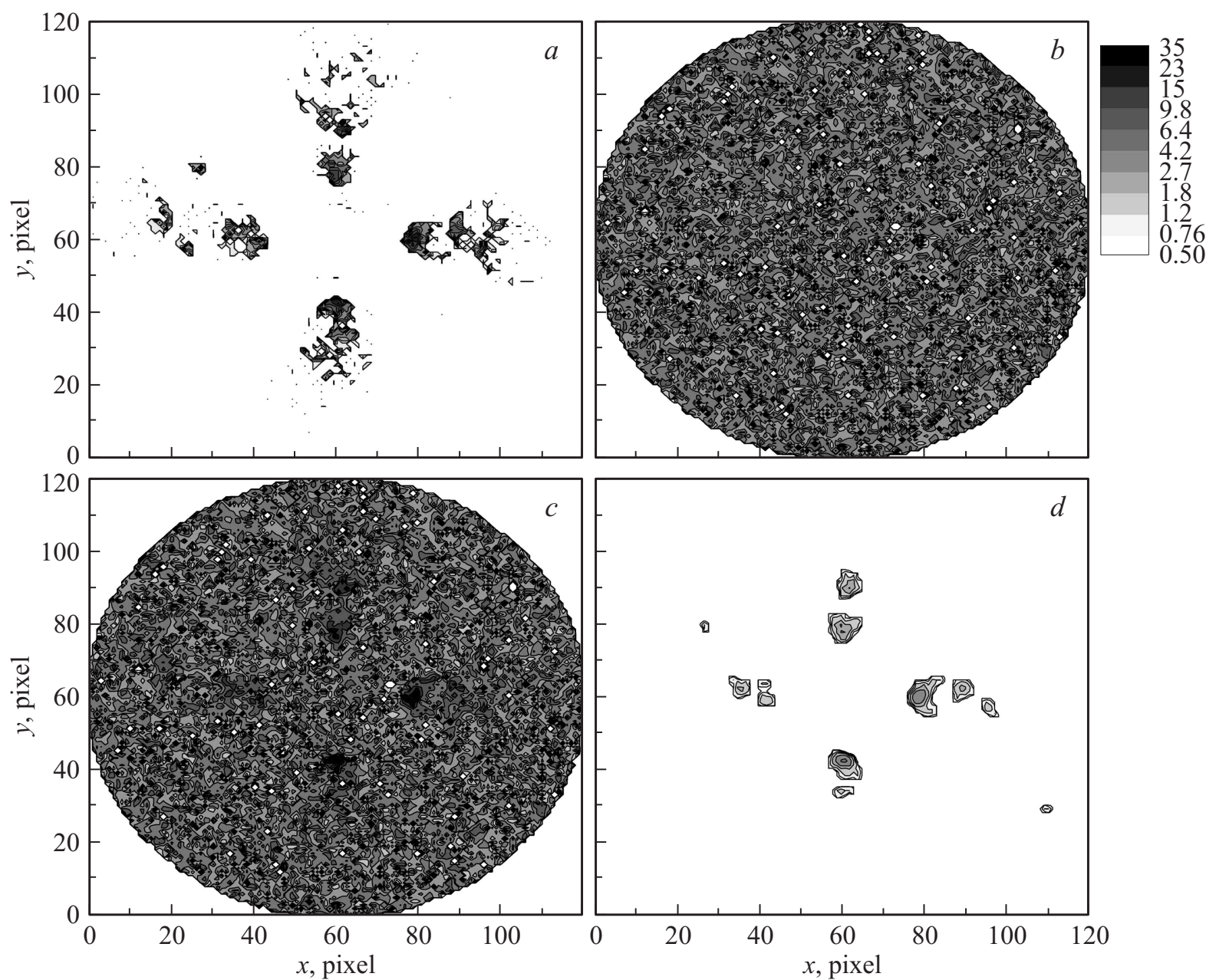

Рис. 5. Изображения в фокальных плоскостях телескопов (для удобства восприятия фокальные плоскости всех телескопов приведены на одном рисунке): $a$ - черенковская вспышка, сформировавшаяся при взаимодействии гамма-кванта (энергией $5 \mathrm{GeV})$ с атмосферой; $b-$ оптический фон ночного неба; $c-$ совместное изображение $a$ и $b ; d-$ изображение $c$ после обработки фильтром. Максимальные значения интенсивностей составляют 30 фотонов/пиксел $(a)$, 18 фотонов/пиксел $(b)$, 35 фотонов/пиксел $(c), 5$ единиц/пиксел $(d)$. Для всех изображений справедлива единая цветовая шкала, показанная справа от изображения $(b)$.

неба и кривыми чувствительности МЛФД производства Hamamatsu и ФТИ им. А.Ф. Иоффе.

2) Разработан оригинальный код для моделирования оптической системы телескопа обсерватории ALEGRO, позволяющий изменять параметры телескопа (диаметр зеркала, фокусное расстояние и т.д.) и учитывать различные варианты размещения телескопов обсерватории на местности.

3) Разработан алгоритм и создан численный код для очистки изображений, формирующихся в фокальных плоскостях телескопов ALEGRO, от оптических фотонов фона ночного неба. На рис. 5 представлены результаты моделирования и обработки модельных наблюдательных данных: для наглядности на рис. 5, $a$ представлено изображение в фокальных плоскостях исходной черенковской вспышки (без какого-либо влияния оптического фона), показанной на рис. 3; на рис. $5, b$ представлено изображение оптического фо- на ночного неба с экспозицией $10 \mathrm{~ns}$ для потока $4.6 \cdot 10^{12}$ photon $\mathrm{m}^{-2} \mathrm{~s}^{-1} \mathrm{ster}^{-1}[138]$; на рис. 5, с представлено совместное изображение, т. е. модель реального оптического сигнала, поступающего на блок фотодетекторов; на рис. $5, d$ представлен конечный результат обработки симулированных зашумленных данных. При моделировании была рассмотрена регистрирующая система из четырех идентичных телескопов с параболическими зеркалами диаметром $30 \mathrm{~m}$, фокусным расстоянием $46.9 \mathrm{~m}$ и полем зрения $2.9 \mathrm{deg}$, расположенных в точках с координатами $(100 \mathrm{~m}, 0 \mathrm{~m}) ;(0 \mathrm{~m}, 100 \mathrm{~m})$; $(-100 \mathrm{~m}, 0 \mathrm{~m})$ и $(0 \mathrm{~m},-100 \mathrm{~m})$ на высоте $5 \mathrm{~km}$ над уровнем моря. Поверхность блока оптического приемника в рассматриваемом случае моделируется как круглая область диаметром $1.2 \mathrm{~m}$, разбитая на 11310 pixel (форма пиксела - квадрат со стороной $1 \mathrm{~cm}$ ).

Интенсивность засветки пиксела $(k-$ номер пиксела), которая определяет яркость пикселов на 


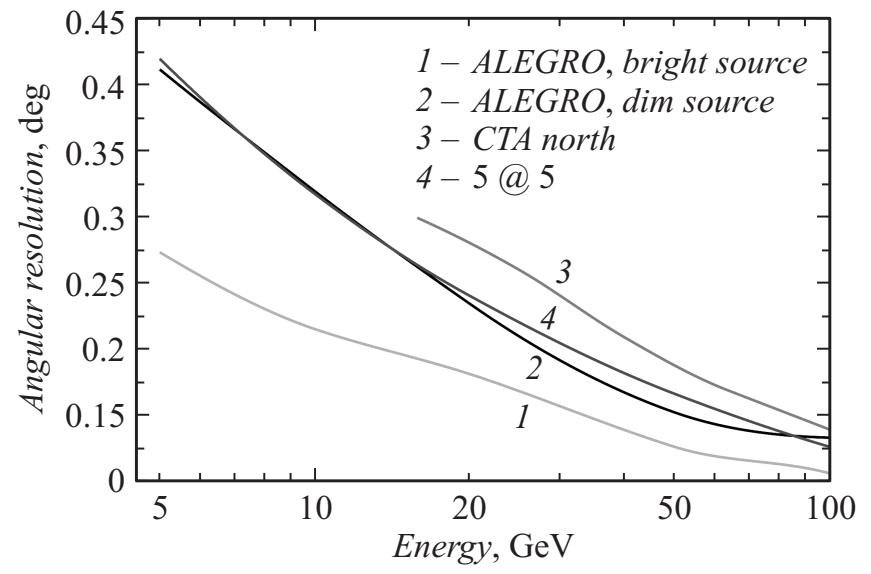

Рис. 6. Модельное угловое разрешение обсерваторий ALEGRO, CTA и 5@5. Кривая 2 соответствует разрешению обсерватории ALEGRO при наблюдении слабых источников (расчет выполнен для условий, использованных в работе [7], посвященной проекту 5@5), а кривая 1 - разрешению при наблюдении достаточно ярких источников, которые могут быть детектированы при использовании жестких критериев отбора событий (выбраковка до 85\% событий с энергией $10 \mathrm{GeV}$ ).

рис. 5, $a-c$, - это количество фотонов (натуральное число), попавших в пиксел в течение временного промежутка характерной длительности $10 \mathrm{~ns}$. Эффективность детектирования фотона при моделировании задавалась как случайная величина с нормальным распределением с параметрами $0.30 \pm 0.07$ электрон/фотон. Учетом эффективности детектирования фотонов и особенностями математической обработки сигнала, согласно разработанному алгоритму, объясняется разница в размерности и амплитуде величин, приведенных на рис. 5, с и $5, d$. Таким образом, рис. 5 иллюстрирует принципиальную возможность надежной регистрации гамма-квантов с энергиями около $5 \mathrm{GeV}$ наземными черенковскими гамма-обсерваториями. Ожидаемое угловое разрешение на энергии $5 \mathrm{GeV}$ составляет $0.3-0.4 \mathrm{deg}$ в зависимости от критериев отбора событий (рис. 6). Такое разрешение достаточно для разделения переменных гаммаисточников в диапазоне около $5 \mathrm{GeV}$. Измерение кривых блеска переменных гамма-источников в этом диапазоне является одной из основных задач проекта ALEGRO.

4) Разработан оригинальный код для определения параметров Хилласа черенковской вспышки. Этот код позволяет определять направление прихода первичной частицы в каждом конкретном случае, а также путем проведения серии численных экспериментов по восстановлению характеристик первичных частиц позволяет оценить параметры гамма-обсерватории (угловое и энергетическое разрешение) в целом.

Разработанные модули пакета ALEGRO Soft для моделирования ШАЛ и обработки наблюдательных данных позволили изучить ряд научных проблем, возникших при планировании наземной гамма-обсерватории нового поколения с чувствительностью в диапазоне энергий от
$5 \mathrm{GeV}$ : показать наличие „тяжелого хвоста“ в распределении ППЧФ (т.е., отсутствие конечного среднеквадратичного отклонения ППЧФ в области ствола ШАЛ) при низких энергиях первичных гамма-квантов [130], оценить влияние оптического фона ночного неба на возможности черенковского телескопа четвертого поколения по регистрации космических гамма-квантов с энергиями ниже $10 \mathrm{GeV}$ и наблюдению гамма-вспышек [141] и показать возможность такой регистрации.

Эффективность разработанного метода определения характеристик первичных частиц и основанного на нем пакета кодов ALEGRO Soft оценена путем проведения численных экспериментов по моделированию широких атмосферных ливней от частиц заданных параметров с помощью симулирующей части пакета кодов с последующим восстановлением характеристик первичных частиц (типа частиц, энергии, направления прихода) с помощью аналитической части пакета. Для диапазона энергий 5-100 GeV проведено свыше 70000 численных экспериментов. Результаты проведенных исследований показывают, что разработанный метод (и базирующийся на нем пакет кодов) будет эффективным инструментом, позволяющим обрабатывать наблюдательные данные черенковских наземных гамма-обсерваторий и не уступающим мировым аналогам (методам и кодам для обработки таких данных), а на низких энергиях $(5-30 \mathrm{GeV})$ даже превосходящим их. Пример обработки наблюдательных данных с помощью пакета кодов ALEGRO Soft приведен на рис. 7. Полученные результаты позволяют ожидать, что при соответствующем уровне разработки аппаратной части наземного черенковского гаммателескопа в наблюдениях достаточно ярких космических гамма-источников на энергиях около $20 \mathrm{GeV}$ может быть достигнуто угловое разрешение около $0.16 \mathrm{deg}$ (рис. 7), что примерно в полтора раза лучше, чем ожидаемые значения углового разрешения, полученные при моделировании в рамках других планируемых проектов, например, проектов СТА (хуже $0.25 \mathrm{deg}$ на энергиях менее $25 \mathrm{GeV}$ [114]) или 5@5 (около $0.24 \mathrm{deg}$ на энергии $20 \mathrm{GeV}$ [7]). Такое угловое разрешение может быть достигнуто путем выбора достаточно жестких критериев качества изображений, используемых для анализа: в исследуемом случае для анализа было отобрано лишь около $8 \%$ изображений. Несмотря на то что при таком подходе подавляющее большинство изображений не используется для построения карты исследуемой области небесной сферы и определения угловых координат источника, абсолютное количество обрабатываемых событий от гамма-квантов с энергией $20 \mathrm{GeV}$ будет больше чем, например, общее количество событий от гамма-квантов с энергией $100 \mathrm{GeV}$, что говорит о том, что значительная статистическая значимость может быть достигнута при разумных временах наблюдения даже при использовании жестких критериев отбора и отбраковке более 90\% изображений. Следует отметить, что при измерении иных характеристик гамма-источника 

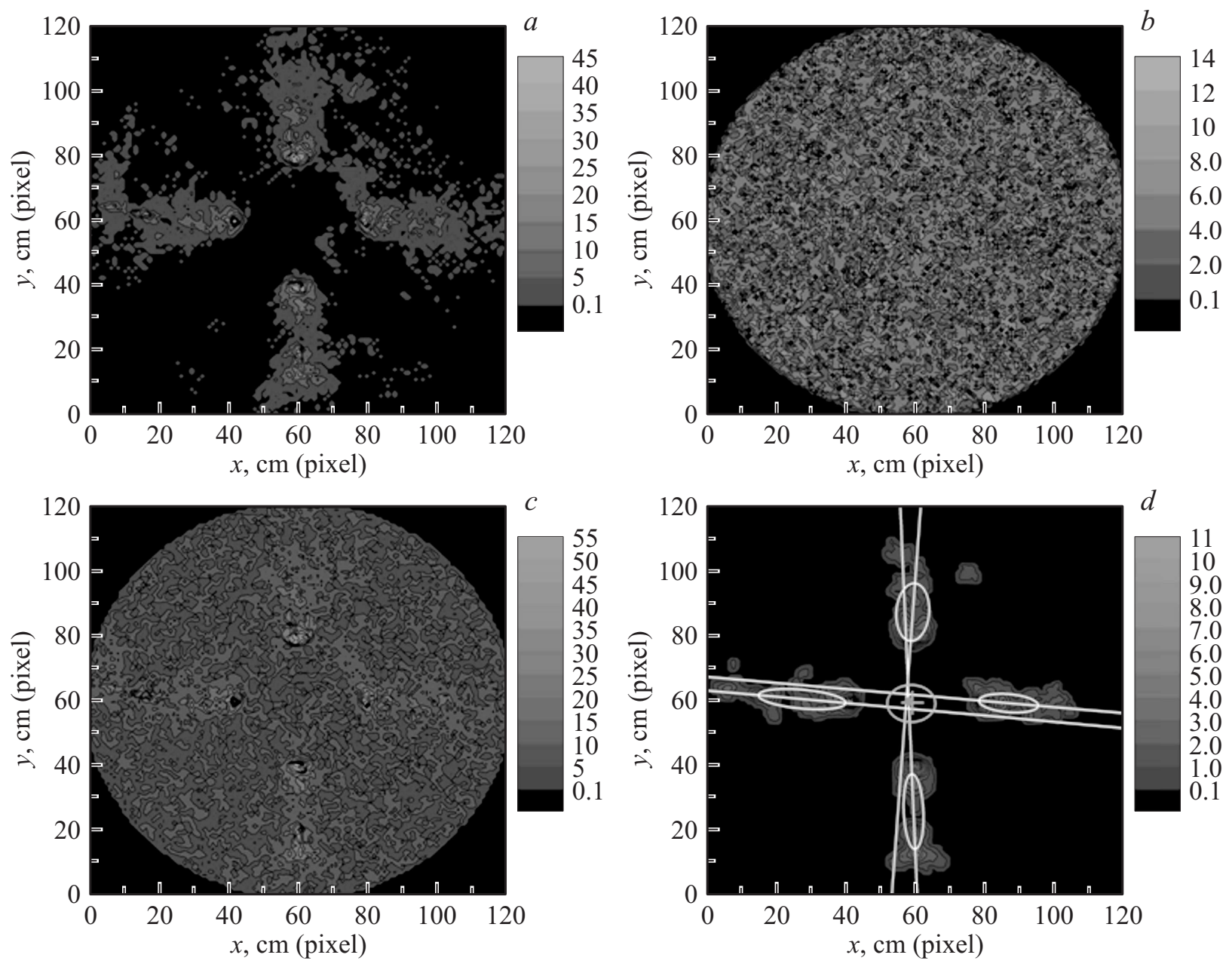

Рис. 7. $a-$ изображения в фокальных плоскостях телескопа черенковской вспышки, сформировавшейся при взаимодействии вертикально падающего гамма-кванта с энергией $20 \mathrm{GeV}$ с атмосферой (получено при моделировании ШАЛ с помощью симулирующей части пакета ALEGRO Soft). Для наглядности изображения в фокальных плоскостях всех телескопов приведены на одном рисунке. $b$ - изображение, формирующееся оптическим фоном ночного неба в фокальной плоскости телескопа за $10 \mathrm{~ns}$ (получено с помощью симулятора оптического фона ночного неба пакета ALEGRO Soft); $c$ - совместное изображение черенковской вспышки и оптического фона - модель реального сигнала, регистрируемого гамма-обсерваторией. 8 - изображение после обработки аналитической частью пакета кодов ALEGRO Soft: произведена очистка от оптического шума и анализ параметров Хилласа для определения направления прихода первичной частицы. Светлые эллипсы - фитирующие эллипсы Хилласа, направление больших главных осей которых (светлые прямые) показывает направление развития ШАЛ в атмосфере. Точка в центре рисунка показывает восстановленное направление прихода первичного гамма-кванта, вычисленное по усредненному значению координат пересечений светлых прямых. Крест в центре показывает координаты истинного направления прихода первичного гамма-кванта. Эллипс в центре - контур доверительного определения направления прихода первичной частицы по уровню одно стандартное отклонение, определяющий угловое разрешение телескопа при данном алгоритме обработки. Размер большой полуоси эллипса в центре соответствует примерно $0.16 \mathrm{deg}$. Полное поле зрения телескопов обсерватории $2.9 \mathrm{deg}$ градуса соответствует 120 пикселам.

(в частности, спектральных) могут быть выбраны другие критерии отбраковки, что позволит использовать в анализе более значительную долю снятых изображений (вплоть до 100\%). Модельные кривые углового разрешения обсерватории ALEGRO для различных условий наблюдения приведены на рис. 6. Для сравнения на том же рисунке дана кривая углового разрешения СТА [114] при наблюдении слабых источников (т.е. для случая оптимизации чувствительности).
Проведенные численные расчеты (в частности, данные работы [130]) позволяют оценить энергетическое разрешение обсерватории ALEGRO в общем случае (т.е. при усреднении по значениям прицельного параметра падающих гамма-квантов) на уровне $\Delta E / E \sim 0.8$ на энергии $5 \mathrm{GeV}$ и $\Delta E / E \sim 0.2$ на энергии $100 \mathrm{GeV}$, что не уступает планируемому энергетическому разрешению обсерватории СТА North [114]. На рис. 8 приведена модельная кривая, описывающая верхний (наихуд- 


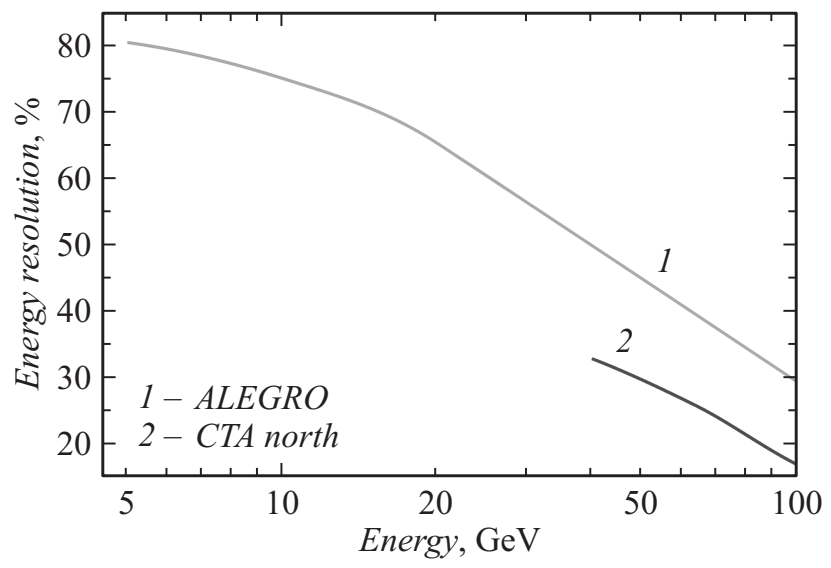

Рис. 8. Модельное энергетическое разрешение обсерваторий ALEGRO и CTA.

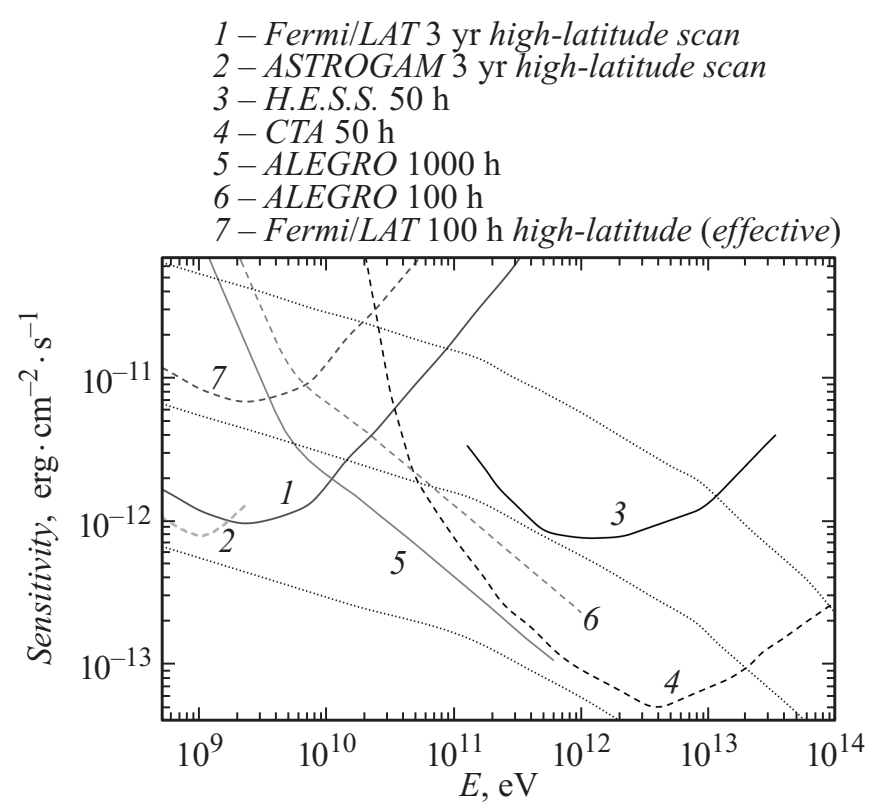

Рис. 9. Чувствительность действующих и проектируемых инструментов к потокам гамма-излучения от точечных космических источников. Более длительные по сравнению с другими черенковскими гамма-телескопами экспозиции ALEGRO связаны с планируемым увеличением рабочего цикла этой обсерватории за счет работы в лунные ночи.

ший, наиболее консервативный) предел энергетического разрешения обсерватории ALEGRO, соответствующий наблюдению гамма-квантов с прицельным параметром $r=0 \mathrm{~m}$ относительно центра обсерватории. Для сравнения на том же рисунке дана кривая энергетического разрешения СТА в общем случае (усредненная по прицельному параметру) [114]. Следует отметить, что эти характеристики обсерватории ALEGRO могут быть улучшены с разработкой более совершенных методов обработки данных, основанных как на массированном моделировании, так и на анализе реальных наблюдательных данных в процессе эксплуатации.
Оценка чувствительности обсерватории ALEGRO показывает, что в основном диапазоне 5-50 GeV она будет иметь чувствительность к потокам от точечных гамма-источников на уровне $10^{-11}-10^{-12} \mathrm{TeV} \mathrm{cm}^{-2} \mathrm{~s}^{-1}$ (рис. 9). При коротких экспозициях чувствительность ALEGRO будет превосходить чувствительность Fermi/LAT и CTA в диапазоне $20-50 \mathrm{GeV}$, а в диапазонах 5-20 и 80-100 GeV будет сопоставима с чувствительностью указанных инструментов.

\section{7. Конструкторские разработки в рамках проекта ALEGRO}

К настоящему времени в рамках проекта ALEGRO проведен ряд изыскательских и конструкторских работ.

В лабораториях СПбПУ и ФТИ им. А.Ф. Иоффе изготовлен прототип субблока черенковского детектора из 64 пикселей на основе лавинных фотодиодов Hamamatsu Photonics s10362-33. Планируется, что в окончательном варианте детектор одного телескопа из массива ALEGRO будет состоять из примерно 10000 пикселей-фотодиодов производства Hamamatsu Photonics, SensL и/или собственного производства ФТИ им. А.Ф. Иоффе. Субблок оптического приемника предназначается для использования в составе черенковского гамма-телескопа и является законченным функциональным узлом для построения многоэлементного оптического приемника. Для обработки информации, поступающей с субблока (количества попадающих на него фотонов, координат - адресов сработавших ячеек), разработана система регистрации и сбора данных (read-out). Она обеспечивает регистрацию сигналов от лавинных фотодиодов, их усиление, предварительную обработку, сбор и передачу данных с последующей обработкой на программном уровне в режиме реального времени.

В ФТИ им. А.Ф. Иоффе проводится разработка оригинальных лавинных фотодиодов $[142,143]$ для решения специальных задач черенковской гамма-астрономии, в частности, определения типа первичной частицы посредством наблюдения УФ-составляющей черенковского излучения (см., например, [144]) и расширения возможностей обсерватории (проведения наблюдений в лунные ночи [145] и др.). Достигнуты значения эффективности детектирования около 2\% в далеком УФ диапазоне [142]. Ведется работа по увеличению эффективности детектирования и расширению диапазона спектральной чувствительности в видимую часть спектра.

\section{Заключение}

Наблюдения излучения экстремальных космических объектов методами черенковской гамма-астрономии необходимы для решения ряда актуальных задач современной астрофизики и фундаментальной физики. Перспективная черенковская гамма-обсерватория четвертого поколения ALEGRO позволит осуществлять 
чувствительные наблюдения в важном, но до сих пор мало исследованном диапазоне энергий 5-50 GeV.

К настоящему времени в рамках проекта ALEGRO получены следующие результаты, определяющие основные параметры будущей обсерватории.

1. Регистрация космических гамма-квантов с энергией $5 \mathrm{GeV}$ может быть осуществлена с помощью системы оптических телескопов с диаметром зеркала $30 \mathrm{~m}$, установленной на высоте $4-5 \mathrm{~km}$ над уровнем моря. При размещении на высоте $5 \mathrm{~km}$ гамма-обсерватория будет иметь как минимум в полтора раза более низкую пороговую энергию наблюдений, чем при размещении на высоте $2 \mathrm{~km}$ при прочих равных условиях.

2. Влияние оптического фона ночного неба существенно для процесса регистрации космических гаммаквантов в диапазоне $1-10 \mathrm{GeV}$, но еще не является непреодолимым природным препятствием для регистрации космического гамма-излучения. В частности, вероятность регистрации гамма-кванта с энергией $3 \mathrm{GeV}$ (без сохранения информации об энергии и направлении прихода, что достаточно для измерения кривых блеска гамма-всплесков) составляет около 50\% при малых зенитных углах наблюдения $(<10 \mathrm{deg})$ и малых прицельных параметрах (< 100 m относительно геометрического центра обсерватории в конфигурации „квадрат“ со стороной $140 \mathrm{~m}$ (см. также [141])).

3. Ожидаемое энергетическое разрешение обсерватории ALEGRO составляет $\Delta E / E \sim 0.8$ на энергии $5 \mathrm{GeV}$ (см. также [130]) и $\Delta E / E \sim 0.2$ на энергии $100 \mathrm{GeV}$.

4. Путем выбора критериев отбора анализируемых изображений может быть достигнуто угловое разрешение $0.16 \mathrm{deg}$ на энергии $20 \mathrm{GeV}$ при малых зенитных углах наблюдения.

5. Чувствительность обсерватории ALEGRO в диапазоне 5-100 GeV может составлять $10^{-11}-10^{-12} \mathrm{TeV} \cdot \mathrm{cm}^{-2} \mathrm{~s}^{-1}$.

Таким образом, результаты моделирования и опытных конструкторских разработок показывают, что обсерватория ALEGRO будет эффективным инструментом для наблюдений космических источников гамма-излучения в диапазоне энергий 5-50 GeV. Эта обсерватория позволит решить ряд актуальных задач экспериментальной гамма-астрономии и астрофизики высоких энергий.

Работа Е.Е. Холупенко, А.М. Красильщикова и П.Н. Аруева частично поддержана грантом РФФИ ОФИ-м 16-29-13009.

\section{Список литературы}

[1] Aharonian F. Very High Energy Cosmic Gamma Radiation: a Crucial Window on the Extreme Universe. World Scientific Publishing Co. Pte. Ltd., 2004. 350 p.

[2] Brazier K.T.S., Carraminana A., Chadwick P.M. et al. // Nucl. Phys. B. Proc. Suppl. 1990. Vol. 14. N 1. P. 188-190.

[3] Zych A.D., Ait-Ouamer F., Kerrick A.D. et al. // Bull. Am. Astron. Soc. 1990. Vol. 22. P. 1222.
[4] Ahnen M.L., Ansoldi S., Antonelli L.A. et al. // ApJL. 2015. Vol. 815. N 2. P. L23.

[5] Abramowski A., Acero F., Aharonian F. et al. // PRL. 2011. Vol. 106. N 16. P. 161301.

[6] Amelino-Camelia G., Ellis J., Mavromatos N.E., Nanopoulos D.V., Sarkar S. // Nature. 1998. N 6687. P. 763-765.

[7] Aharonian F.A., Konopelko A.K., V"olk H.J., Quintana H. // Astropart. Phys. 2001. Vol. 15. N 4. P. 335-356.

[8] Hillas A.M. // Astropart. Phys. 2013. Vol. 43. P. 19-43.

[9] Galbraith W., Jelley J.V. // Nature. 1953. Vol. 171. N 4347. P. 349-350.

[10] Nesterova N.M., Chudakov A.E. // JETP. 1955. Vol. 28. P. $388-389$.

[11] Chudakov A.E., Dadykin V.L., Zatsepin V.I., Nesterova N.M. // Proceedings of the 8th ICRC. 1963. Vol. 4. P. 199-204.

[12] Jelley J.V., Porter N.A. // Q. J. R. Astron. Soc. 1963. Vol. 4. P. 275-293.

[13] Fazio G.G., Helmken H.F., Rieke G.H., Weekes T.C. // ApJ. 1968. Vol. 154. P. L83.

[14] Fegan D.J., Mc Breen B., O'Mongain E.P., Porter N.A., Slevin P.J. // Can. J. Phys. 1968. Vol. 46. P. S433-S436.

[15] Charman W.N., Fruin J.H., Jelley J.V., Fegan D.J. et al. // Proc. 11th Int. Cosmic-Ray Conf. Budapest. 1970. P. 59-63.

[16] Stepanian A.A., Vladimirskii B.M., Neshpor Iu.I., Fomin V.P. // ApSS. 1975. Vol. 38. P. 267-282.

[17] Li T.-P., Ma Y.-Q. // ApJ. 1983. Vol. 272. P. 317-324.

[18] Gould R.J., Burbidge G.R. // Ann. Astrophys. 1965. Vol. 28. P. 171-201.

[19] Apparao M.V. Krishna. // Nature. 1969. Vol. 223. N 5204. P. $385-386$.

[20] Fazio G.G., Helmken H.F., Rieke G.H., Weekes T.C. // Proc. 11th Int. Cosmic-Ray Conf. Budapest. 1970. P. 115-118.

[21] Vladimirsky B.M., Zyskin Yu.L., Neshpor Yu.I., Stepanian A.A., Fomin V.P., Shitov V.G. // Proc. Very High Energy Gamma-Ray Astr. Conf. Crimea. 1991. P. 21.

[22] Cawley M.F., Fegan D.J., Gibbs K. et al. // Proc. 19th Int. Cosmic-Ray Conf. La Jolla. 1985. Vol. 3. P. 453-456.

[23] Hillas A.M. // Proc. 19th Int. Cosmic-Ray Conf. (La Jolla). 1985. Vol. 3. P. 445-448.

[24] Aharonian F.A., Akhperjanian A.G., Kankanian A.S. et al. // Proc. 22nd Internat. Cosmic Ray Conf. (Dublin). 1991. Vol. 2. P. 615-617.

[25] Aharonian F., Akhperjanian A., Beilicke M. et al. // ApJ. 2004. Vol. 614. N 2. P. 897-913.

[26] Aharonian F., Akhperjanian A., Barrio J. et al. // A\&A. 1997. Vol. 327. P. L5-L8.

[27] Barrau A., Bazer-Bachi R., Beyer E. et al. // Nucl. Instr. Methods Phys. Res. A. 1998. Vol. 416. N 2-3. P. 278-292.

[28] Ebisuzaki S., Edwards P.G., Fujii H. et al. // Proc. 22nd Internat. Cosmic Ray Conf. Dublin. 1991. Vol. 2. P. 607-610.

[29] Tsuchiya K., Enomoto R., Ksenofontov L.T. et al. // ApJ. 2004. Vol. 606. N 2. P. L115-L118.

[30] Bhatt N., Agarwal N.K., Bhat C.K. et al. // Proc. 26th Int. Cosmic Ray Conf. Salt Lake City. 1999. Vol. 4. P. 104-107.

[31] Chadwick P.M., Dipper N.A., Dowthwaite J.C. et al. // Proc. 19th Int. Cosmic-Ray Conf. La Jolla. 1985. Vol. 3. P. 406-409.

[32] Armstrong P., Chadwick P.M., Cottle P.J. et al. // Exper. Astr. 1999. Vol. 9. N 2. P. $51-80$.

[33] Sinitsyna V.G., Nikolsky S.I., Alaverdian A.Y. et al. // Proc. 24th Int. Cosmic Ray Conf. Rome. 1995. P. 334-337. 
[34] Weekes T.C., Cawley M.F., Fegan D.J. et al. // ApJ. 1989. Vol. 342. P. 379-395.

[35] Punch M., Akerlof C.W., Cawley M.F. et al. // Nature. 1992. Vol. 358. N 6386. P. 477-478.

[36] Piron F. // Proc. 26th Int. Cosmic Ray Conf. Salt Lake City. 1999. Vol. 3. P. 326.

[37] Андреева Н.А., Зыскин Ю.Л., Калекин О.Р., Нешпор Ю.И., Степанян А.А., Фомин В.П., Чаленко Н.Н., Шитов В.Г. // ПАЖ. 2000. Т. 26. С. 243-248.

[38] Hofmann $W$. // Proc. 26th Int. Cosmic Ray Conf. Trukuba. 2003. P. 2811-2814.

[39] Aharonian F., Akhperjanian A.G., Aye K.-M. et al. // A\&A. 2004. Vol. 425. P. L13-L17.

[40] Baixeras C. // Nucl. Phys. B. Proc. Suppl. 2003. Vol. 114. P. 247-252.

[41] Cortina J. // ApSS. 2005. Vol. 297. N 1-4. P. 245-255.

[42] Holder J. // Proc. 29th Int. Cosmic Ray Conf. Pune. 2005. Vol. 5. P. 379-382.

[43] Holder J., Acciari V.A., Aliu E. et al. // AIP Conf. Proc. 2008. Vol. 1085. P. 657-660.

[44] Weekes T.C., Badran H., Biller S.D. et al. // Astropart. Phys. 2002. Vol. 17. N 2. P. 221-243.

[45] Cortina J., Goebel F., Schweizer T. // arXiv preprint arXiv:0907.1211.

[46] Moralejo A. // arXiv preprint arXiv:0912.3673.

[47] Nakajima D., Fink D., Hose J. et al. // Proc. 33rd Int. Cosmic Ray Conf. Rio De Janeiro. 2013. P. L0787.

[48] Aleksić J., Alvarez E.A., Antonelli L.A. et al. // Astropart. Phys. 2012. Vol. 35. N 7. P. 435-448.

[49] Becherini Y., Punch M. // AIP Conf. Proc. 2012. Vol. 1505. P. 741-744.

[50] Renaud M., Vink J., Decourchelle A. // ApJ. 2006. Vol. 647. N 1. P. L41-L44.

[51] Prantzos N., Boehm C., Bykov A.M. et al. // Rev. Mod. Phys. 2011. Vol. 83. N 3. P. $1001-1056$.

[52] Churazov E., Sunyaev R., Isern J. et al. // Nature. 2014. Vol. 12. N 7515. P. 406-408.

[53] Aharonian F., Akhperjanian A.G., Bazer-Bachi A.R. et al. // A\&A. 2007. Vol. 464. P. 235-243.

[54] Bykov A.M., Chevalier R.A., Ellison D.C., Uvarov Yu.A. // ApJ. 2000. Vol. 538. N 1. P. 203-216.

[55] Ellison D.C., Slane P., Patnaude D.J., Bykov A.M. // ApJ. 2012. Vol. 744. N 1. P. 39.

[56] Slane P., Bykov A.M., Ellison D.C., Dubner G., Castro D. // Space Sci. Rev. 2015. Vol. 188. N 1-4. P. 187-210.

[57] Bykov A.M. // Space Sci. Rev. 2001. Vol. 99. N 1-4. P. 317-326.

[58] Ackermann M., Ajello M., Allafort A. et al. // Science. 2011. Vol. 334. N 6059. P. 1103-1107.

[59] Bykov A.M. // Space Sci. Rev. 2014. Vol. 22. P. 77.

[60] Abramowski A., Acero F., Aharonian F. et al. // A\&A. 2012. Vol. 537. P. A114.

[61] Bykov A.M., Ellison D.C., Gladilin P.E., Osipov S.M. // MNRAS. 2015. Vol. 453. N 1. P. 113-121.

[62] Abdo A.A., Ackermann M., Ajello M. et al. // ApJ. 2010. Vol. 708. N 2. P. $1254-1267$.

[63] Aleksić J., Alvarez E.A., Antonelli L.A. et al. // ApJ. 2011. Vol. 742. N 1. P. 43.

[64] Abramowski A., Aharonian F., Ait Benkhali F. et al. // A\&A. 2014. Vol. 562. P. L4.

[65] Stegmann C. // Докл. на конф. Astroparticle Physics 2014-A joint TeVPA / iDM Conf. 2014. Amsterdam. https://www.mpi-hd.mpg.de/hfm/HESS/201406-TeVPAAmsterdam-nofilm.pdf
[66] Chrétien M., Bolmont J., Jacholkowska A. arXiv preprint arXiv:1509.03545.

[67] Ansoldi S., Antonelli L.A., Antoranz P. et al. // A\&A. 2016. Vol. 585. P. A133.

[68] Richards G.T. // arXiv preprint arXiv:1508.06969.

[69] Abdo A.A., Ackermann M., Ajello M. et al. // Science. 2011. Vol. 331. N 6018. P. 739.

[70] Bykov A.M., Pavlov G.G., Artemyev A.V., Uvarov Yu.A. // MNRAS Lett. 2012. Vol. 421. N 1. P. L67-L71.

[71] Aleksić J., Ansoldi S., Antonelli L.A. et al. // A\&A. 2014. Vol. 567. P. L8.

[72] Aliu E., Archambault S., Arlen T. et al. // ApJ. 2013. Vol. 764. N 1. P. 38.

[73] Abramowski A., Acero F., Aharonian F. et al. // A\&A. 2012. Vol. 548. P. A38.

[74] Abramowski A., Acero F., Aharonian F. et al. // A\&A. 2012. Vol. 545. P. L2.

[75] Albert J., Aliu E., Anderhub H. et al. // Science. 2006. Vol. 312. N 5781. P. 1771-1773.

[76] Aharonian F., Akhperjanian A.G., Bazer-Bachi A.R. et al. // A\&A. 2006. Vol. 460. N 3. P. 743-749.

[77] Acciari V.A., Beilicke M., Blaylock G. et al. // ApJ. 2008. Vol. 679. N 2. P. 1427-1432.

[78] Rico J., Rissi M., Bordas P. et al. // Proc. 30th Int. Cosmic Ray Conf. Mérida. 2007. Vol. 2. P. 699-702.

[79] Worrall D.M., Birkinshaw M., Remillard R.A. et al. // ApJ. 1999. Vol. 516. N 1. P. 163-176.

[80] Lindfors E. // JPCS. 2012. Vol. 355. N 1. P. 012003.

[81] Benbow $W$. arXiv preprint arXiv:1508.07251.

[82] Abramowski A., Aharonian F., Ait Benkhali F. et al. // A\&A. 2014. Vol. 564. P. A9.

[83] Abramowski A., Aharonian F., Ait Benkhali F. et al. // Nature. 2016. Vol. 531. N 7595. P. 476-479.

[84] Toma K., Yamazaki R., Nakamura T. // ApJ. 2005. Vol. 620. N 2. P. $835-839$.

[85] Svinkin D.S., Frederiks D.D., Aptekar R.L. et al. // ApJS. 2016. Vol. 224. N 1. P. 10.

[86] Atwood W.B., Abdo A.A., Ackermann M. et al. // ApJ. 2009. Vol. 697. N 2. P. $1071-1102$.

[87] Abramowski A., Aharonian F., Ait Benkhali F. et al. // A\&A. 2014. Vol. 565. P. A16.

[88] Aleksić J., Ansoldi S., Antonelli L.A. et al. // MNRAS. 2014. Vol. 437. N 4. P. 3103-3111.

[89] Aliu E., Aune T., Barnacka A. et al. // ApJL. 2014. Vol. 795. N 1. P. L3.

[90] Bykov A., Gehrels N., Krawczynski H., Lemoine M., Pelletier G., Pohl M. // Space Sci. Rev. 2012. Vol. 173. N 1-4. P. 309-339.

[91] Abazajian K.N., Agrawal P., Chacko Z., Kilic C. // Phys. Rev. D. 2012. Vol. 85. N 12. P. 123543.

[92] Tempel E., Hektor A., Raidal M. // J. Cosmol. Astropart. Phys. 2012. N 09. P. 032.

[93] Arlen T., Aune T., Beilicke M. // ApJ. 2012. Vol. 757. N 2. P. 123.

[94] Abramowski A., Aharonian F., Ait Benkhali F. et al. // Phys. Rev. D. 2014. Vol. 90. N 11. P. 112012.

[95] Ahnen M.L., Ansoldi S., Antonelli L.A. et al. // J. Cosmol. Astropart. Phys. 2016. N 02. P. 039.

[96] Zitzer B. arXiv preprint arXiv:1509.01105.

[97] Bröggen M., Bykov A., Ryu D., Röttgering H. // Space Sci. Rev. 2012. Vol. 166. N 1-4. P. 187-213.

[98] Никишов А.И. // ЖЭТФ. 1961. Т. 41. С. 549-550.

[99] Costamante L. // Int. J. Mod. Phys. 2013. Vol. 22. N 13. P. 1330025. 
[100] Krennrich F., Dwek E., Imran A. // ApJ. 2008. Vol. 689. P. L93.

[101] Protheroe R.J., Meyer H. // Phys. Rew. Lett. B. 2000. Vol. 493. P. 1-6.

[102] Horns D., Meyer M. // JCAP. 2012. N 2. P. 033.

[103] Rubtsov G.I., Troitsky S.V. // Pis'ma v ZhETF. 2014. Vol. 100. N 6. P. 397-401.

[104] De Angelis A., Mansutti O., Roncadelli M. // Phys. Lett. 2008. Vol. 659. N 5. P. 847-855.

[105] Simet M., Hooper D., Serpico P. // Phys. Rev. D. 2008. Vol. 77. P. 063001.

[106] Fairbairn M., Rashba T., Troitsky S. // Phys. Rev. D. 2011. Vol. 84. P. 125019.

[107] Jaeckel J., Ringwald A. // Ann. Rev. Nucl. Part. Sci. 2010. Vol. 60. P. 405-437.

[108] Troitsky S. // Phys. Rev. D. 2016. Vol. 93. N 4. P. 045014.

[109] Онлайн каталог космических источников тераэлектроновольтного гамма-излучения. Электронный ресурс. Режим доступа: http://tevcat.uchicago.edu/

[110] Mazin D. // AIP Conf. Proc. 2009. Vol. 1112. P. 111-120.

[111] Aharonian F., Akhperjanian A.G., Barres de Almeida U. et al. // Phys. Rev. Let. 2008. Vol. 101. N 26. P. 261104.

[112] Zitzer B. arXiv preprint arXiv:1307.8382.

[113] Albert J., Aliu E., Anderhub H. et al. // Phys. Rev. Let. B. 2008. Vol. 668. N 4. P. 253-257.

[114] Cherenkov Telescope Array. https://portal.cta-observatory.org/Pages/Home.aspx

[115] Barres de Almeida U. // Astron. Nachr. 2015. Vol. 336. N 8-9. P. 795-799.

[116] Arons J. // ApJ. 1983. Vol. 266. P. 215-241.

[117] Chiang J., Romani R.W. // ApJ. 1994. Vol. 436. N 2. P. 754-761.

[118] Ruderman M. // Proc. Symp. Pulsars: 13 years of research on neutron stars / eds. W. Sieber \& R. Wielebinski D. Reidel Publishing Co., Dordrecht. 1981. P. 87-97.

[119] Yadigaroglu I.A., Romani R.W. // ApJ. 1995. Vol. 449. P. $211-215$.

[120] Grenier I.A. // A\&A. 2000. Vol. 364. P. L93-L96.

[121] Aharonian F.A., Bogovalov S.V. // New Astron. 2003. Vol. 8. N 2. P. $85-103$.

[122] Harding A. // AIP Conf. Proc. 2001. Vol. 558. P. 115-126.

[123] Bosch-Ramon V. // Mem. S.A.It. 2012. Vol. 83. P. 194-201.

[124] Koul R., Rannot R.C., Mitra A. et al. // Proc. 32rd Int. Cosmic Ray Conf. Beijing. 2011. Vol. 9. P. 107-111.

[125] Yadav K. Докл. на конф. „Very High Energy Phenomena in the Universe, Rencontres de Moriond“, 2013. La Thuile, Электронный ресурс. Режим доступа: https://moriond.in2p3.fr/J13/transparencies/yadav.pdf

[126] Major Atmospheric Cherenkov Telescope Experiment (MACE). Электронный ресурс. Режим доступа: http://www.barc.gov.in/pg/nrl-harl/mace.html

[127] Tümer O.T., Kerrick A.D., O'Neill T.J., White R.S., Zych A.D. // Proc. 22nd Internat. Cosmic Ray Conf. Dublin. 1991. Vol. 2. P. 634-637.

[128] Profumo S., Kamionkowski M. // J. Cosmol. Astropart. Phys. 2003. N 03. P. 003.

[129] Frach T. // J. Instrum. 2012. Vol. 7. N 01. P. C01112.

[130] Васильев Г.И., Холупенко Е.Е., Байко Д.А. и др. // НТВ СПбГПУ. Физико-математические науки. 2011. № 4. C. $79-86$.

[131] Korpar S., Adachi I., Chagani H. et al. // Nucl. Phys. B Proc. Suppl. 2009. Vol. 197. N 1. P. 283-287.

[132] Lightfoot P.K., Barker G.J., Mavrokoridis K. et al. // J. Instrum. 2008. Vol. 10. N 10. P. 001.
[133] Bretz T., Anderhub H., Backes M. et al. arXiv preprint arXiv:1403.3573.

[134] Spargo J. // National Radio Astronomy Observatory Observer. 1980. Vol. 21. N 1. P. 39-42.

[135] New space telescope begins to comb the cosmos. Электронный ресурс. Режим доступа:

http://www.spacedaily.com/reports/New_space_telescope_ begins_to_comb_the_cosmos_999.html

[136] Agostinelli S., Allison J., Amako K. et al. // Nucl. Instrum. Method. Phys. Res. A. 2003. Vol. 506. P. 250-303.

[137] Официальный веб-сайт пакета программ CORSIKA. Электронный ресурс. Режим доступа: https://www.ikp.kit.edu/corsika

[138] Oña-Wilhelmi E., Cortina J., de Jager O.C., Fonseca V. // Astropart. Phys. 2004. Vol. 22. N 1. P. 95-102.

[139] Leinert Ch., Bowyer S., Haikala L.K. et al. // A\&ASS. 1998. Vol. 127. P. 1-99.

[140] Официальный веб-сайт HAMAMATSU Photonics. Электронный ресурс. Режим доступа: http://www.datasheetlib.com/datasheet/707358/s10362-33_ hamamatsu-photonics.html

[141] Васильев Г.И., Холупенко Е.Е., Яблоков С.Н., Байко Д.А., Быков А.М., Красильщиков А.М., Павлов Г.Г. // НТВ СПбГПУ. Физико-математические науки. 2012. № 3. C. 191.

[142] Забродский В.В., Аруев П.Н., Белик В.П. и др. // Письма в ЖТФ. 2014. Т. 40. Вып. 8. С. 23-29.

[143] Zabrodskii V., Aruev P., Belik V. et al. // Nucl. Instrum. Method. Phys. Res. A. 2015. Vol. 787. P. 348-352.

[144] Kalekin O.R., Neshpor Yu.I., Stepanyan A.A. et al. // Astr. Lett. 1995. Vol. 21. N 2. P. 163-167.

[145] Chantell M.C., Sarazin X., Fleury P. et al. // Proc. 24th Int. Cosmic Ray Conf. Rome. 1995. Vol. 2. P. 560-563. 